\title{
A nonlocal parabolic model for type-I superconductors
}

\author{
M. Slodička, K. Van Bockstal \\ Research Group NaM², Department of Mathematical Analysis, Ghent University, Galglaan 2, 9000 Ghent, Belgium
}

\begin{abstract}
A vectorial nonlocal linear parabolic problem on a bounded domain with applications in superconductors of type-I is studied. The nonlocal term is represented by a (space) convolution with a singular kernel (arising in Eringen's model). The well-posedness of the problem is discussed under low regularity assumptions and the error estimates for an implicit and semi-implicit time-discrete scheme (based on backward Euler approximation) are established. It is shown that the solution of the problem satisfies a simpler nonlocal problem with a positive definite kernel if the normal component of the unknown vector field equals zero on the boundary of the domain. Numerical experiments support the obtained theoretical results.
\end{abstract}

Keywords: nonlocal superconductors, singular convolution kernel, time discretization, error estimates 2010 MSC: 35Q61, 65M20, 82D55

\section{Introduction}

Although a large number of studies have been devoted to the microscopic theory of superconductivity (the first microscopic theory was the BCS theory by Bardeen, Cooper and Schrieffer [1]), the macroscopic theory seems to have received relatively little attention in the literature. Nonetheless, since the discovery of high-temperature superconductors in 1986 [2], industrial applications require macroscopic models and their mathematical analysis for superconductivity. Superconductors can be classified into two other main types, namely type-I and type-II superconductors. What first follows is a brief overview of the available models for type-II superconductors. One of the first macroscopic models was Bean's critical-state model [3]. This model is studied by many authors [4, 5, 6, 7]. Another model used in the modelling of type-II superconductors is the power law constitutive relation by Rhyner [8]. This relation in combination with Maxwell's equations is investigated in $[9,10,11]$. In this contribution, a nonlocal macroscopic problem for type-I superconductors in terms of the magnetic field is proposed and analysed, based on the theory by Pippard and Eringen [12, 13]. Recent engineering applications about nonlocal effects in type-I superconductors can be found in [14, 15, 16, 17].

The starting point of the modelling part of this contribution is the phenomenological theory of superconductivity by London and London in $1935[18,19]$. They explained that a macroscopic description of type-I superconductors involves a two-fluid model. One fluid consists of normal electrons and the other one of superconducting electrons. Superconducting electrons cross the metal without suffering any resistance, in contrast to electrons in a normal material, which scatter and suffer resistance to their motion. Below the critical temperature $T_{c}$, when the superconductive material loses all resistivity, the current consists of superconducting electrons and normal electrons. Above the critical temperature only normal electrons occur. Accordingly, the current density $\boldsymbol{J}$ is supposed to be the sum of a normal and superconducting part, that is

$$
\boldsymbol{J}=\boldsymbol{J}_{n}+\boldsymbol{J}_{s}
$$

Email addresses: Marian.Slodicka@UGent. be (M. Slodička), Karel.VanBockstal@UGent.be (K. Van Bockstal)

URL: http://cage.UGent.be/〜ms / (M. Slodička), http://cage.UGent.be/〜 kvb/ (K. Van Bockstal) 
The superconductive material occupies a bounded domain $\Omega \subset \mathbb{R}^{3}$ with a Lipschitz continuous boundary $\partial \Omega$. The symbol $\boldsymbol{\nu}$ denotes the outward unit normal vector on $\partial \Omega$. To derive a mathematical model for type-I superconductors, the eddy current version of the Maxwell's equations (the displacement current is neglected in Ampère's law) is used. They can be written as

$$
\begin{aligned}
& \nabla \times \boldsymbol{H}=\boldsymbol{J}, \\
& \text { Ampère's law } \\
& \nabla \times \boldsymbol{E}=-\partial_{t} \boldsymbol{B} . \\
& \text { Faraday's law }
\end{aligned}
$$

Note that this approximation is valid in highly conductive media [20,21]. Moreover, a linear dependence of the magnetic induction $\boldsymbol{B}$ on the magnetic field $\boldsymbol{H}$ is assumed, namely

$$
\boldsymbol{B}=\mu \boldsymbol{H}
$$

where the constant $\mu>0$ stands for the magnetic permeability of the material. Applying the divergence operator to the Faraday law and integrating in time gives $\nabla \cdot \boldsymbol{B}(t)=\nabla \cdot \boldsymbol{B}(t=0)$. Therefore, assuming $\nabla \cdot \boldsymbol{B}(t=0)=0$, it is ensured that the magnetic induction remains divergence free for any time. The normal density current $\boldsymbol{J}_{n}$ is required to satisfy Ohm's law, $\boldsymbol{J}_{n}=\sigma \boldsymbol{E}, \sigma>0$ being the conductivity of normal electrons and $\boldsymbol{E}$ the electric field. Due to the previous considerations, the eddy current model can be rewritten in the following form

$$
\begin{aligned}
\nabla \times \boldsymbol{H} & =\sigma \boldsymbol{E}+\boldsymbol{J}_{s}, \\
\nabla \times \boldsymbol{E} & =-\mu \partial_{t} \boldsymbol{H} .
\end{aligned}
$$

London and London postulated two equations, in addition to Maxwells equations, governing the electromagnetic field in a superconductor [18]:

$$
\partial_{t} \boldsymbol{J}_{s}=\Lambda^{-1} \boldsymbol{E} \quad \text { and } \quad \nabla \times \boldsymbol{J}_{s}=-\Lambda^{-1} \boldsymbol{B},
$$

where $\Lambda=\frac{m_{e}}{n_{s} e^{2}}$, with $n_{s}$ the number of superelectrons per unit volume, $m_{e}$ and $-e$ the mass and the electric charge of an electron respectively. These equations provide a correct description of two basic properties of superconductors: perfect conductivity and perfect diamagnetism (Meissner effect) [19]. Since $\nabla \cdot \boldsymbol{B}=0$, there exists a magnetic vector potential $\boldsymbol{A}$ such that $\boldsymbol{B}=\nabla \times \boldsymbol{A}$ and $\nabla \cdot \boldsymbol{A}=0$, cf. [22]. If the domain $\Omega$ is simply connected, $\boldsymbol{A}$ is uniquely determined when $\boldsymbol{A} \cdot \boldsymbol{\nu}=0$ on $\partial \Omega$, this is the London gauge. Therefore, the second London equation can be rewritten in the local form

$$
\boldsymbol{J}_{s}=-\Lambda^{-1} \boldsymbol{A} .
$$

The local theory of London and London is generalized to nonlocal theories, for instance by Pippard and Eringen. In 1953, Pippard [12] proposed following modification of the local expression (1.3)

$$
\boldsymbol{J}_{s, p}(\boldsymbol{x}, t)=\int_{\Omega} Q\left(\boldsymbol{x}-\boldsymbol{x}^{\prime}\right) \boldsymbol{A}\left(\boldsymbol{x}^{\prime}, t\right) \mathrm{d} \boldsymbol{x}^{\prime}, \quad(\boldsymbol{x}, t) \in Q_{T}:=\Omega \times(0, T)
$$

with

$$
Q\left(\boldsymbol{x}-\boldsymbol{x}^{\prime}\right) \boldsymbol{A}\left(\boldsymbol{x}^{\prime}, t\right)=-\widetilde{C} \frac{\boldsymbol{x}-\boldsymbol{x}^{\prime}}{\left|\boldsymbol{x}-\boldsymbol{x}^{\prime}\right|^{4}}\left[\boldsymbol{A}\left(\boldsymbol{x}^{\prime}, t\right) \cdot\left(\boldsymbol{x}-\boldsymbol{x}^{\prime}\right)\right] \exp \left(-\frac{\left|\boldsymbol{x}-\boldsymbol{x}^{\prime}\right|}{r_{0}}\right),
$$

where $\widetilde{C}:=\frac{3}{4 \pi \xi_{0} \Lambda}>0$. The length $\xi_{0}$ is called the coherence length of the material. The points which contribute to the integral are separated by distances of order $r_{0}$ or less, with $r_{0}$ defined by

$$
\frac{1}{r_{0}}=\frac{1}{\xi_{0}}+\frac{1}{l}
$$

with $l$ the mean free path of the electrons in the material. This nonlocal expression is based on Chambers nonlocal Ohm's law. However, Pippard's nonlocal law fails to explain the vanishing of electrical 
resistance. For this reason, the nonlocal representation of the superconductive current by Eringen [13] is considered (1984). This representation identifies the state of the superconductor, at time $t$, with the field $\boldsymbol{H}(\cdot, t)$ and is given by the linear functional

$$
\boldsymbol{J}_{s, e}(\boldsymbol{x}, t)=\int_{\Omega} \sigma_{0}\left(\left|\boldsymbol{x}-\boldsymbol{x}^{\prime}\right|\right)\left(\boldsymbol{x}-\boldsymbol{x}^{\prime}\right) \times \boldsymbol{H}\left(\boldsymbol{x}^{\prime}, t\right) \mathrm{d} \boldsymbol{x}^{\prime}=:-\left(\mathcal{K}_{0} \star \boldsymbol{H}\right)(\boldsymbol{x}, t), \quad(\boldsymbol{x}, t) \in Q_{T},
$$

where $\sigma_{0}:(0, \infty) \rightarrow \mathbb{R}$ is defined by

$$
\sigma_{0}(s)= \begin{cases}\frac{\widetilde{C}}{2 s^{2}} \exp \left(-\frac{s}{r_{0}}\right) & s<r_{0} \\ 0 & s \geqslant r_{0} .\end{cases}
$$

The integral is to be taken over the whole volume of the metal. The dependence of $\boldsymbol{J}_{s}$ on time $t$ is solely through $\boldsymbol{H}$. The function $\sigma_{0}$ becomes unbounded for $\boldsymbol{x}^{\prime}=\boldsymbol{x}$. Moreover, $\sigma_{0}$ is so chosen that it is possible to recover from (1.4) the London equations and the form given by Pippard [13, 19]. Consequently, the form by Eringen is a more direct generalizaton of the London theory in comparison with Pippard's nonlocal law. Throughout the paper, the nonlocal law of Eringen is considered and is denoted by $\boldsymbol{J}_{s}$ instead of $\boldsymbol{J}_{s, e}$. Taking the curl of (1.2) results into the following parabolic integrodifferential equation

$$
\sigma \mu \partial_{t} \boldsymbol{H}+\nabla \times \nabla \times \boldsymbol{H}+\nabla \times\left(\mathcal{K}_{0} \star \boldsymbol{H}\right)=\mathbf{0} .
$$

Mathematical analysis of integro-differential equations arising from the nonlocal theory of superconductivity has been analysed for smooth electromagnetic fields in [23]. The model considered there was written in terms of the vector potential of $\boldsymbol{H}$. The analysis was based on the spectral analysis and expansion in terms of eigenfunctions. In this contribution, a variational approach is proposed, which can be applied to general geometries without knowledge of the spectrum. Theorems with low assumptions of regularity of a solution are derived. Various numerical schemes for time discretization are designed and the error estimates for approximations are derived. A finite element algorithm is developed and it is programmed using the finite element library DOLFIN from the FEniCS project. A Fredholm type integro-differential equation in 1D based on Pippard's nonlocal superconductivity model is studied in detail in [24] using the Galerkin finite element method. However, in this paper, a 3D model is investigated.

The remainder of this paper is organized as follows. Section 2 summarizes the mathematical tools. Problem (1.6) is presented into detail in Section 3 and the well-posedness of the problem is shown in Section 4. A time-discrete numerical scheme is developed. The existence of a weak solution for each time step is shown. Also the convergence of the method is discussed and error estimates are derived. A modified scheme is considered in Section 5. In Section 6, a new convolution kernel is derived under an additional assumption. The positive definiteness of this kernel is shown. Using the obtained expression, it is demonstrated that the solution of the original model satisfies a simpler equation, which is described and analysed in Subsection 6.2. A comparison with the London equations is given into Section 6.4. Finally, some numerical experiments are developed in Section 7.

\section{Functional setting}

First, some standard notations are introduced. The euclidian norm of a vector $\boldsymbol{v}$ in $\mathbb{R}^{3}$ is expressed by $|\boldsymbol{v}|$. The Lebesgue spaces of vector-valued functions with componentwise $p$-th power integrable functions are denoted by $\mathbf{L}^{p}(\Omega)=\left(L^{p}(\Omega)\right)^{3}$ with the usual norm $\|\cdot\|_{p}$. For instance, in the special case $p=2$, the $\mathbf{L}^{2}(\Omega)$ scalar product is denoted by $(\boldsymbol{u}, \boldsymbol{v})=\int_{\Omega} \boldsymbol{u} \cdot \boldsymbol{v} \mathrm{d} \boldsymbol{x}$ and the corresponding norm is $\|\boldsymbol{v}\|=\sqrt{(\boldsymbol{v}, \boldsymbol{v})}$. The following spaces are used in our analysis: $\mathbf{H}^{1}(\Omega), \mathbf{H}^{2}(\Omega), \mathbf{H}(\mathbf{c u r l}, \Omega)$ and the fractional Sobolev spaces $\mathbf{H}^{s}(\Omega)$ - see [22]. The spaces $\mathbf{H}^{1}(\Omega), \mathbf{H}^{2}(\Omega)$, and $\mathbf{H}(\mathbf{c u r l}, \Omega)$ are 
respectively endowed with the norms

$$
\begin{aligned}
\|\varphi\|_{\mathbf{H}^{1}(\Omega)}^{2} & =\|\varphi\|^{2}+\|\nabla \varphi\|^{2}, \\
\|\varphi\|_{\mathbf{H}^{2}(\Omega)}^{2} & =\|\varphi\|_{\mathbf{H}^{1}(\Omega)}^{2}+\|\Delta \varphi\|^{2}, \\
\|\varphi\|_{\mathbf{H}(\mathbf{c u r l}, \Omega)}^{2} & =\|\boldsymbol{\varphi}\|^{2}+\|\nabla \times \varphi\|^{2} .
\end{aligned}
$$

Further, the space of test functions will be $\mathbf{H}_{0}^{1}(\Omega)$ and $\mathbf{H}_{0}(\operatorname{curl}, \Omega)$ with the inherited norms $\|\boldsymbol{\varphi}\|_{\mathbf{H}^{1}(\Omega)}$ and $\|\varphi\|_{\mathbf{H}(\mathbf{c u r l}, \Omega)}$, respectively. Its dual spaces are denoted by $\mathbf{H}^{-1}(\Omega)$ and $\mathbf{H}_{0}^{-1}(\mathbf{c u r l}, \Omega)$ respectively. The following Friedrichs inequality holds true for every $\varphi \in \mathbf{H}_{0}^{1}(\Omega)$

$$
\|\varphi\|_{\mathbf{H}_{0}^{1}(\Omega)}^{2} \leqslant C\|\nabla \varphi\| .
$$

The space $\operatorname{Lip}\left([0, T], \mathbf{L}^{2}(\Omega)\right)$ is the space of Lipschitz continuous functions $\boldsymbol{F}:[0, T] \rightarrow \mathbf{L}^{2}(\Omega)$. The values $C, \varepsilon$ and $C_{\varepsilon}$ are generic and positive constants independent of the discretization parameter $\tau$. The value $\varepsilon$ is small and $C_{\varepsilon}=C\left(\varepsilon^{-1}\right)$. To reduce the number of arbitrary constants, the notation $a \lesssim b$ is used if there exists a constant $C$ such that $a \leqslant C b$. Finally, Abel's summation rule is recalled:

$$
2 \sum_{i=1}^{n}\left(a_{i}-a_{i-1}\right) a_{i}=a_{n}^{2}-a_{0}^{2}+\sum_{i=1}^{n}\left(a_{i}-a_{i-1}\right)^{2}, \quad a_{i} \in \mathbb{R} .
$$

\section{Parabolic nonlocal problem for superconductivity}

For ease of exposition, set $\mu=\sigma=1$ in (1.6). Also a possible source term $\boldsymbol{F}$ is considered in the right-hand side (RHS). The aim of this paper is to address the well-posedness of the following problem

$$
\left\{\begin{aligned}
\partial_{t} \boldsymbol{H}+\nabla \times \nabla \times \boldsymbol{H}+\nabla \times\left(\mathcal{K}_{0} \star \boldsymbol{H}\right) & =\boldsymbol{F} & & \text { in } Q_{T} ; \\
\boldsymbol{H} \times \boldsymbol{\nu} & =\mathbf{0} & & \text { on } \partial \Omega \times(0, T) ; \\
\boldsymbol{H}(\boldsymbol{x}, 0) & =\boldsymbol{H}_{0} & & \text { in } \Omega ;
\end{aligned}\right.
$$

to design a numerical scheme for computations and to derive error estimates for the time discretization. To obtain the magnetic boundary condition in (3.1), it is assumed that the magnetic field outside the domain $\Omega$ equals zero [22, p. 8]. However, from mathematical viewpoint, it is also possible to consider a boundary condition of the form $\boldsymbol{H} \times \boldsymbol{\nu}=\boldsymbol{g}$. This is not done to increase the readability of the text. Instead of the eddy current approximation of the Maxwell equations, it is also possible to consider the full Maxwell system. Then, a fully hyperbolic problem should be considered instead of (3.1) (cf. [25, Chapter 5]). This is done in [26]. The mathematics of eddy-current approximation has recently been developed in some other settings, see for instance [27, 28, 29]. The main difference in the analysis of problem (3.1), in comparison with the available results, is caused by the nonlocal term in (3.1). Let us also note that the vectorial field $\sigma_{0}(|\boldsymbol{x}|) \boldsymbol{x}$ belongs to $\mathbf{L}^{p}(\Omega)$ for $1 \leqslant p<3$. Therefore, it is easily checked that

$$
\left|\boldsymbol{J}_{s}(\boldsymbol{x})\right|=\left|\left(\mathcal{K}_{0} \star \boldsymbol{H}\right)(\boldsymbol{x})\right| \leqslant C(p)\|\boldsymbol{H}\|_{p}, \quad p>\frac{3}{2}, \quad \forall \boldsymbol{x} \in \Omega .
$$

The variational formulation of (3.1) reads as: find $\boldsymbol{H}(t) \in \mathbf{H}_{0}(\mathbf{c u r l}, \Omega)$ with $\partial_{t} \boldsymbol{H}(t) \in \mathbf{H}_{0}^{-1}(\operatorname{curl}, \Omega)$ such that

$$
\left(\partial_{t} \boldsymbol{H}(t), \boldsymbol{\varphi}\right)+(\nabla \times \boldsymbol{H}(t), \nabla \times \boldsymbol{\varphi})+\left(\mathcal{K}_{0} \star \boldsymbol{H}(t), \nabla \times \boldsymbol{\varphi}\right)=(\boldsymbol{F}(t), \boldsymbol{\varphi}), \quad \forall \boldsymbol{\varphi} \in \mathbf{H}_{0}(\mathbf{c u r l}, \Omega),
$$

for a.e $t \in[0, T]$.

Theorem 1 (Uniqueness). The problem (3.1) admits at most one solution $\boldsymbol{H} \in C\left([0, T], \mathbf{L}^{2}(\Omega)\right) \cap$ $L^{2}\left((0, T), \mathbf{H}_{0}(\operatorname{curl}, \Omega)\right)$. 
Proof: Assume that we have two solutions $\boldsymbol{H}_{1}, \boldsymbol{H}_{2}$. Then $\boldsymbol{H}=\boldsymbol{H}_{1}-\boldsymbol{H}_{2}$ fulfills (3.1) with $\boldsymbol{H}_{0}=$ $\mathbf{0}=\boldsymbol{F}$. Setting $\boldsymbol{\varphi}=\boldsymbol{H}$ into (3.3) and integrating in time for $t \in(0, T)$, we find that

$$
\frac{1}{2}\|\boldsymbol{H}(t)\|^{2}+\int_{0}^{t}\|\nabla \times \boldsymbol{H}\|^{2}+\int_{0}^{t}\left(\mathcal{K}_{0} \star \boldsymbol{H}, \nabla \times \boldsymbol{H}\right)=0 .
$$

Using the Cauchy and Young inequalities together with (3.2) for $p=2$ to the last term, we arrive at

$$
\|\boldsymbol{H}(t)\|^{2}+\int_{0}^{t}\|\nabla \times \boldsymbol{H}\|^{2} \leqslant \varepsilon \int_{0}^{t}\|\nabla \times \boldsymbol{H}\|^{2}+C_{\varepsilon} \int_{0}^{t}\|\boldsymbol{H}\|^{2} .
$$

Fixing a sufficiently small positive $\varepsilon$ and applying the Grönwall argument, we get that $\boldsymbol{H}=\mathbf{0}$ a.e. in $Q_{T}$.

The following theorem describes the natural stability of the solution $\boldsymbol{H}$ of (3.1).

Theorem 2 (Stability). Suppose $\boldsymbol{F} \in L^{2}\left((0, T), \mathbf{L}^{2}(\Omega)\right)$.

(i) If $\boldsymbol{H}_{0} \in \mathbf{L}^{2}(\Omega)$ then

$$
\max _{t \in[0, T]}\|\boldsymbol{H}(t)\|^{2}+\int_{0}^{T}\|\nabla \times \boldsymbol{H}\|^{2} \leqslant C .
$$

(ii) If $\nabla \cdot \boldsymbol{F}=0=\nabla \cdot \boldsymbol{H}_{0}$ then $\nabla \cdot \boldsymbol{H}(t)=0$ for any $t \in[0, T]$. Moreover, we have that

$$
\int_{0}^{T}\left\|\partial_{t} \boldsymbol{H}\right\|_{\mathbf{H}_{0}^{-1}(\operatorname{curl}, \Omega)}^{2} \leqslant C .
$$

(iii) If $\boldsymbol{H}_{0} \in \mathbf{H}_{0}(\mathbf{c u r l}, \Omega)$ then

$$
\max _{t \in[0, T]}\|\nabla \times \boldsymbol{H}(t)\|^{2}+\int_{0}^{T}\left\|\partial_{t} \boldsymbol{H}\right\|^{2} \leqslant C
$$

(iv) If $\boldsymbol{F}(0) \in \mathbf{L}^{2}(\Omega), \partial_{t} \boldsymbol{F} \in L^{2}\left((0, T), \mathbf{L}^{2}(\Omega)\right), \nabla \times\left(\mathcal{K}_{0} \star \boldsymbol{H}_{0}\right) \in \mathbf{L}^{2}(\Omega), \boldsymbol{H}_{0} \in \mathbf{H}_{0}(\mathbf{c u r l}, \Omega)$ and $\nabla \times \nabla \times \boldsymbol{H}_{0} \in \mathbf{L}^{2}(\Omega)$ then

$$
\max _{t \in[0, T]}\left\|\partial_{t} \boldsymbol{H}(t)\right\|^{2}+\int_{0}^{T}\left\|\nabla \times \partial_{t} \boldsymbol{H}\right\|^{2} \leqslant C .
$$

Proof: (i) Setting $\boldsymbol{\varphi}=\boldsymbol{H}$ in (3.3) and integrating in time for $t \in(0, T)$, we get

$$
\frac{1}{2}\|\boldsymbol{H}(t)\|^{2}+\int_{0}^{t}\|\nabla \times \boldsymbol{H}\|^{2}=\frac{1}{2}\left\|\boldsymbol{H}_{0}\right\|^{2}+\int_{0}^{t}(\boldsymbol{F}, \boldsymbol{H})-\int_{0}^{t}\left(\mathcal{K}_{0} \star \boldsymbol{H}, \nabla \times \boldsymbol{H}\right) .
$$

Using the Cauchy and Young inequalities to the RHS together with (3.2) for $p=2$, we obtain

$$
\|\boldsymbol{H}(t)\|^{2}+\int_{0}^{t}\|\nabla \times \boldsymbol{H}\|^{2} \leqslant C+C_{\varepsilon} \int_{0}^{t}\|\boldsymbol{H}\|^{2}+\varepsilon \int_{0}^{t}\|\nabla \times \boldsymbol{H}\|^{2} .
$$

We obtain the desired result choosing a sufficiently small positive $\varepsilon$ and involving the Grönwall argument.

(ii) Take the divergence of (3.1) and integrate in time to arrive at $\nabla \cdot \boldsymbol{H}(t)=\nabla \cdot \boldsymbol{H}_{0}=0$ for all $t \in[0, T]$. We rewrite (3.3) as follows

$$
\left(\partial_{t} \boldsymbol{H}, \boldsymbol{\varphi}\right)=(\boldsymbol{F}, \boldsymbol{\varphi})-(\nabla \times \boldsymbol{H}, \nabla \times \boldsymbol{\varphi})-\left(\mathcal{K}_{0} \star \boldsymbol{H}, \nabla \times \boldsymbol{\varphi}\right), \quad \boldsymbol{\varphi} \in \mathbf{H}_{0}(\operatorname{curl}, \Omega) .
$$


A simple calculation implies

$$
|(\boldsymbol{F}, \boldsymbol{\varphi})| \leqslant\|\boldsymbol{F}\|\|\boldsymbol{\varphi}\|, \quad|(\nabla \times \boldsymbol{H}, \nabla \times \boldsymbol{\varphi})| \leqslant\|\nabla \times \boldsymbol{H}\|\|\nabla \times \varphi\|
$$

and

$$
\left|\left(\mathcal{K}_{0} \star H, \nabla \times \varphi\right)\right| \stackrel{(3.2)}{\lesssim}\|\boldsymbol{H}\|\|\nabla \times \varphi\|
$$

Remember that

$$
\left\|\partial_{t} \boldsymbol{H}\right\|_{\mathbf{H}_{0}^{-1}(\mathbf{c u r l}, \Omega)}=\sup _{\boldsymbol{\varphi} \in \mathbf{H}_{0}(\mathbf{c u r l}, \Omega)} \frac{\left(\partial_{t} \boldsymbol{H}, \boldsymbol{\varphi}\right)}{\|\boldsymbol{\varphi}\|_{\mathbf{H}_{0}(\mathbf{c u r l}, \Omega)}} .
$$

Therefore, using $(i)$, we deduce that

$$
\int_{0}^{T}\left\|\partial_{t} \boldsymbol{H}\right\|_{\mathbf{H}_{0}^{-1}(\operatorname{curl}, \Omega)}^{2} \leqslant C .
$$

(iii) Now, we set $\boldsymbol{\varphi}=\partial_{t} \boldsymbol{H}$ in (3.3) and integrate in time for $t \in(0, T)$ to obtain

$$
\int_{0}^{t}\left\|\partial_{t} \boldsymbol{H}\right\|^{2}+\frac{1}{2}\|\nabla \times \boldsymbol{H}\|^{2}=\frac{1}{2}\left\|\nabla \times \boldsymbol{H}_{0}\right\|^{2}+\int_{0}^{t}\left(\boldsymbol{F}, \partial_{t} \boldsymbol{H}\right)-\int_{0}^{t}\left(\mathcal{K}_{0} \star \boldsymbol{H}, \nabla \times \partial_{t} \boldsymbol{H}\right) .
$$

The second term on the RHS can be estimated using the Cauchy and Young inequalities as follows

$$
\int_{0}^{t}\left(\boldsymbol{F}, \partial_{t} \boldsymbol{H}\right) \leqslant \varepsilon \int_{0}^{t}\left\|\partial_{t} \boldsymbol{H}\right\|^{2}+C_{\varepsilon} \int_{0}^{t}\|\boldsymbol{F}\|^{2} \leqslant \varepsilon \int_{0}^{t}\left\|\partial_{t} \boldsymbol{H}\right\|^{2}+C_{\varepsilon} .
$$

Using the integration by parts formula we may write

$$
\begin{aligned}
\int_{0}^{t}\left(\mathcal{K}_{0} \star \boldsymbol{H}, \nabla \times \partial_{t} \boldsymbol{H}\right) & =\left.\left(\mathcal{K}_{0} \star \boldsymbol{H}, \nabla \times \boldsymbol{H}\right)\right|_{0} ^{t}-\int_{0}^{t}\left(\mathcal{K}_{0} \star \partial_{t} \boldsymbol{H}, \nabla \times \boldsymbol{H}\right) \\
& \stackrel{(3.2)}{ }=C+\varepsilon\|\nabla \times \boldsymbol{H}(t)\|^{2}+C_{\varepsilon}\|\boldsymbol{H}(t)\|^{2} \\
& +\varepsilon \int_{0}^{t}\left\|\partial_{t} \boldsymbol{H}\right\|^{2}+C_{\varepsilon} \int_{0}^{t}\|\nabla \times \boldsymbol{H}\|^{2} \\
& \stackrel{(i)}{\leqslant} \varepsilon\|\nabla \times \boldsymbol{H}(t)\|^{2}+\varepsilon \int_{0}^{t}\left\|\partial_{t} \boldsymbol{H}\right\|^{2}+C_{\varepsilon} .
\end{aligned}
$$

Collecting all considerations above and fixing a sufficiently small positive $\varepsilon$, we conclude the proof.

(iv) First, we differentiate (3.3) with respect to the time variable. Then we set $\boldsymbol{\varphi}=\partial_{t} \boldsymbol{H}$ and integrate in time for $t \in(0, T)$ to get

$$
\frac{1}{2}\left\|\partial_{t} \boldsymbol{H}(t)\right\|^{2}+\int_{0}^{t}\left\|\nabla \times \partial_{t} \boldsymbol{H}\right\|^{2}=\frac{1}{2}\left\|\partial_{t} \boldsymbol{H}(0)\right\|^{2}+\int_{0}^{t}\left(\partial_{t} \boldsymbol{F}, \partial_{t} \boldsymbol{H}\right)-\int_{0}^{t}\left(\mathcal{K}_{0} \star \partial_{t} \boldsymbol{H}, \nabla \times \partial_{t} \boldsymbol{H}\right) .
$$

Employing the Cauchy and Young inequalities, (iii) and (3.2) to the RHS, we get

$$
\left\|\partial_{t} \boldsymbol{H}(t)\right\|^{2}+\int_{0}^{t}\left\|\nabla \times \partial_{t} \boldsymbol{H}\right\|^{2} \leqslant C+\left\|\partial_{t} \boldsymbol{H}(0)\right\|^{2}+C_{\varepsilon} \int_{0}^{t}\left\|\partial_{t} \boldsymbol{H}\right\|^{2}+\varepsilon \int_{0}^{t}\left\|\nabla \times \partial_{t} \boldsymbol{H}\right\|^{2} .
$$

Setting a small $\varepsilon$ and applying the Grönwall argument, we arrive at

$$
\max _{t \in[0, T]}\left\|\partial_{t} \boldsymbol{H}(t)\right\|^{2}+\int_{0}^{T}\left\|\nabla \times \partial_{t} \boldsymbol{H}\right\|^{2} \lesssim 1+\left\|\partial_{t} \boldsymbol{H}(0)\right\|^{2} .
$$

It holds

$$
\partial_{t} \boldsymbol{H}(0)=\boldsymbol{F}(0)-\nabla \times \nabla \times \boldsymbol{H}_{0}-\nabla \times\left(\mathcal{K}_{0} \star \boldsymbol{H}_{0}\right) .
$$


Therefore

$$
\left\|\partial_{t} \boldsymbol{H}(0)\right\| \lesssim 1
$$

which concludes the proof.

\section{Existence of a solution}

To address the existence of a solution to (3.1), the semidiscretization in time is employed. This discretization is based on Rothe's method (cf. [30]). The interval $[0, T]$ is divided into $n$ equidistant subintervals $\left[t_{i-1}, t_{i}\right]$ with time step $\tau=\frac{T}{n}$, thus $t_{i}=i \tau, i=0, \ldots, n$. With the standard notation for the discretized fields

$$
\boldsymbol{h}_{i} \approx \boldsymbol{H}\left(t_{i}\right), \quad \delta \boldsymbol{h}_{i}=\frac{\boldsymbol{h}_{i}-\boldsymbol{h}_{i-1}}{\tau},
$$

the following linear recurrent implicit scheme is proposed to approximate the original problem

$$
\left\{\begin{aligned}
\left(\delta \boldsymbol{h}_{i}, \boldsymbol{\varphi}\right)+\left(\nabla \times \boldsymbol{h}_{i}, \nabla \times \boldsymbol{\varphi}\right)+\left(\mathcal{K}_{0} \star \boldsymbol{h}_{i}, \nabla \times \boldsymbol{\varphi}\right) & =\left(\boldsymbol{f}_{i}, \boldsymbol{\varphi}\right), \quad \boldsymbol{\varphi} \in \mathbf{H}_{0}(\operatorname{curl}, \Omega) \\
\boldsymbol{h}_{0} & =\boldsymbol{H}_{0}
\end{aligned}\right.
$$

which is equivalent to

$$
\begin{aligned}
a\left(\boldsymbol{h}_{i}, \boldsymbol{\varphi}\right) & :=\left(\frac{\boldsymbol{h}_{i}}{\tau}, \boldsymbol{\varphi}\right)+\left(\nabla \times \boldsymbol{h}_{i}, \nabla \times \boldsymbol{\varphi}\right)+\left(\mathcal{K}_{0} \star \boldsymbol{h}_{i}, \nabla \times \boldsymbol{\varphi}\right) \\
& =\left(\boldsymbol{f}_{i}, \boldsymbol{\varphi}\right)+\left(\frac{\boldsymbol{h}_{i-1}}{\tau}, \boldsymbol{\varphi}\right)=: f_{i}(\boldsymbol{\varphi}) .
\end{aligned}
$$

It holds

$$
\left(\mathcal{K}_{0} \star \boldsymbol{h}, \nabla \times \boldsymbol{h}\right) \stackrel{(3.2)}{\leqslant} C_{\varepsilon}\|\boldsymbol{h}\|^{2}+\varepsilon\|\nabla \times \boldsymbol{h}\|^{2}, \quad \forall \boldsymbol{h} \in \mathbf{H}_{0}(\operatorname{curl}, \Omega) .
$$

Thus, the bilinear form $a(\boldsymbol{h}, \boldsymbol{\varphi})$ is elliptic and continuous in $\mathbf{H}_{0}(\operatorname{curl}, \Omega)$ for $\tau<\tau_{0}$. If $\boldsymbol{H}_{0} \in \mathbf{L}^{2}(\Omega)$, then the functional $f_{i}(\boldsymbol{\varphi})$ is linear and bounded in $\mathbf{H}_{0}(\operatorname{curl}, \Omega)$ if $\boldsymbol{h}_{i-1} \in \mathbf{L}^{2}(\Omega)$. Therefore, if $\boldsymbol{H}_{0} \in$ $\mathbf{L}^{2}(\Omega)$, applying the Lax-Milgram lemma gives the existence of a unique solution to (4.1) for any $i=$ $1, \ldots, n$.

First, basic stability result for $\boldsymbol{h}_{i}$ are derived. The a priori estimates in part $(i)$ and $(i i)$ in the following theorem will serve as uniform bounds to prove convergence.

Lemma 1 (A priori estimates). Suppose that $\boldsymbol{F} \in L^{2}\left((0, T), \mathbf{L}^{2}(\Omega)\right)$.

(i) Let $\boldsymbol{H}_{0} \in \mathbf{L}^{2}(\Omega)$. Then, there exists a positive constant $C$ such that

$$
\max _{1 \leqslant i \leqslant n}\left\|\boldsymbol{h}_{i}\right\|^{2}+\sum_{i=1}^{n}\left\|\boldsymbol{h}_{i}-\boldsymbol{h}_{i-1}\right\|^{2}+\sum_{i=1}^{n}\left\|\nabla \times \boldsymbol{h}_{i}\right\|^{2} \tau \leqslant C
$$

for all $\tau<\tau_{0}$.

(ii) If $\nabla \cdot \boldsymbol{H}_{0}=0=\nabla \cdot \boldsymbol{F}$ then $\nabla \cdot \boldsymbol{h}_{i}=0$ for all $i=1, \ldots, n$. Moreover, we have that

$$
\tau \sum_{i=1}^{n}\left\|\delta \boldsymbol{h}_{i}\right\|_{\mathbf{H}_{0}^{-1}(\operatorname{curl}, \Omega)}^{2} \leqslant C
$$

for all $\tau<\tau_{0}$.

(iii) If $\boldsymbol{H}_{0} \in \mathbf{H}_{0}(\mathbf{c u r l}, \Omega)$ then

$$
\max _{1 \leqslant i \leqslant n}\left\|\nabla \times \boldsymbol{h}_{i}\right\|^{2}+\sum_{i=1}^{n}\left\|\nabla \times \boldsymbol{h}_{i}-\nabla \times \boldsymbol{h}_{i-1}\right\|^{2}+\sum_{i=1}^{n}\left\|\delta \boldsymbol{h}_{i}\right\|^{2} \tau \leqslant C
$$


for all $\tau<\tau_{0}$.

(iv) If $\boldsymbol{F}(0) \in \mathbf{L}^{2}(\Omega), \partial_{t} \boldsymbol{F} \in L^{2}\left((0, T), \mathbf{L}^{2}(\Omega)\right), \nabla \times\left(\mathcal{K}_{0} \star \boldsymbol{H}_{0}\right) \in \mathbf{L}^{2}(\Omega), \boldsymbol{H}_{0} \in \mathbf{H}_{0}($ curl,$\Omega)$ and $\nabla \times \nabla \times \boldsymbol{H}_{0} \in \mathbf{L}^{2}(\Omega)$ then

$$
\max _{1 \leqslant i \leqslant n}\left\|\delta \boldsymbol{h}_{i}\right\|^{2}+\sum_{i=1}^{n}\left\|\delta \boldsymbol{h}_{i}-\delta \boldsymbol{h}_{i-1}\right\|^{2}+\sum_{i=1}^{n}\left\|\nabla \times \delta \boldsymbol{h}_{i}\right\|^{2} \tau \leqslant C
$$

for all $\tau<\tau_{0}$.

Proof: (i) Setting $\boldsymbol{\varphi}=\boldsymbol{h}_{i}$ in (4.1), multiplying by $\tau$ and summing up for $i=1, \ldots, j$, we have

$$
\sum_{i=1}^{j}\left(\delta \boldsymbol{h}_{i}, \boldsymbol{h}_{i}\right) \tau+\sum_{i=1}^{j}\left\|\nabla \times \boldsymbol{h}_{i}\right\|^{2} \tau+\sum_{i=1}^{j}\left(\mathcal{K}_{0} \star \boldsymbol{h}_{i}, \nabla \times \boldsymbol{h}_{i}\right) \tau=\sum_{i=1}^{j}\left(\boldsymbol{f}_{i}, \boldsymbol{h}_{i}\right) \tau .
$$

For the first term on the left-hand side (LHS), we use Abel's summation rule

$$
2 \sum_{i=1}^{j}\left(\delta \boldsymbol{h}_{i}, \boldsymbol{h}_{i}\right) \tau=\left\|\boldsymbol{h}_{j}\right\|^{2}-\left\|\boldsymbol{H}_{0}\right\|^{2}+\sum_{i=1}^{j}\left\|\boldsymbol{h}_{i}-\boldsymbol{h}_{i-1}\right\|^{2} .
$$

For the third term on the LHS we have using (3.2) that

$$
\left|\sum_{i=1}^{j}\left(\mathcal{K}_{0} \star \boldsymbol{h}_{i}, \nabla \times \boldsymbol{h}_{i}\right) \tau\right| \leqslant \varepsilon \sum_{i=1}^{j}\left\|\nabla \times \boldsymbol{h}_{i}\right\|^{2} \tau+C_{\varepsilon} \sum_{i=1}^{j}\left\|\boldsymbol{h}_{i}\right\|^{2} \tau .
$$

For the RHS, we apply the Cauchy and Young inequalities to get (for a fixed small $\varepsilon$ )

$$
\left\|\boldsymbol{h}_{j}\right\|^{2}+\sum_{i=1}^{j}\left\|\boldsymbol{h}_{i}-\boldsymbol{h}_{i-1}\right\|^{2}+\sum_{i=1}^{j}\left\|\nabla \times \boldsymbol{h}_{i}\right\|^{2} \tau \lesssim 1+\sum_{i=1}^{j}\left\|\boldsymbol{f}_{i}\right\|^{2} \tau+\sum_{i=1}^{j}\left\|\boldsymbol{h}_{i}\right\|^{2} \tau .
$$

Applying the Grönwall argument, we conclude the proof.

(ii) The result can be readily obtained applying the divergence operator to

$$
\delta \boldsymbol{h}_{i}+\nabla \times \nabla \times \boldsymbol{h}_{i}+\nabla \times\left(\mathcal{K}_{0} \star \boldsymbol{h}_{i}\right)=\boldsymbol{f}_{i} .
$$

It holds

$$
\left(\delta \boldsymbol{h}_{i}, \boldsymbol{\varphi}\right)=\left(\boldsymbol{f}_{i}, \boldsymbol{\varphi}\right)-\left(\nabla \times \boldsymbol{h}_{i}, \nabla \times \boldsymbol{\varphi}\right)-\left(\mathcal{K}_{0} \star \boldsymbol{h}_{i}, \nabla \times \boldsymbol{\varphi}\right), \quad \boldsymbol{\varphi} \in \mathbf{H}_{0}(\operatorname{curl}, \Omega) .
$$

Further, we may write

$$
\left|\left(\boldsymbol{f}_{i}, \boldsymbol{\varphi}\right)\right| \leqslant\left\|\boldsymbol{f}_{i}\right\|\|\boldsymbol{\varphi}\|, \quad\left|\left(\nabla \times \boldsymbol{h}_{i}, \nabla \times \boldsymbol{\varphi}\right)\right| \leqslant\left\|\nabla \times \boldsymbol{h}_{i}\right\|\|\nabla \times \boldsymbol{\varphi}\|
$$

and

$$
\left|\left(\mathcal{K}_{0} \star \boldsymbol{h}_{i}, \nabla \times \boldsymbol{\varphi}\right)\right| \stackrel{(3.2)}{\lesssim}\left\|\boldsymbol{h}_{i}\right\|\|\nabla \times \boldsymbol{\varphi}\| .
$$

Thus using

$$
\left\|\delta \boldsymbol{h}_{i}\right\|_{\mathbf{H}_{0}^{-1}(\operatorname{curl}, \Omega)}=\sup _{\boldsymbol{\varphi} \in \mathbf{H}_{0}(\mathbf{c u r l}, \Omega)} \frac{\left(\delta \boldsymbol{h}_{i}, \boldsymbol{\varphi}\right)}{\|\boldsymbol{\varphi}\|_{\mathbf{H}_{0}(\operatorname{curl}, \Omega)}}
$$

and $(i)$, we deduce that

$$
\tau \sum_{i=1}^{n}\left\|\delta \boldsymbol{h}_{i}\right\|_{\mathbf{H}_{0}^{-1}(\operatorname{curl}, \Omega)}^{2} \leqslant C .
$$


(iii) Setting $\boldsymbol{\varphi}=\delta \boldsymbol{h}_{i}$ in (4.1), multiplying by $\tau$ and summing up for $i=1, \ldots, j$, we have

$$
\sum_{i=1}^{j}\left\|\delta \boldsymbol{h}_{i}\right\|^{2} \tau+\sum_{i=1}^{j}\left(\nabla \times \boldsymbol{h}_{i}, \nabla \times \boldsymbol{h}_{i}-\nabla \times \boldsymbol{h}_{i-1}\right)+\sum_{i=1}^{j}\left(\mathcal{K}_{0} \star \boldsymbol{h}_{i}, \nabla \times \delta \boldsymbol{h}_{i}\right) \tau=\sum_{i=1}^{j}\left(\boldsymbol{f}_{i}, \delta \boldsymbol{h}_{i}\right) \tau .
$$

Abel's summation rule helps us to get

$$
2 \sum_{i=1}^{j}\left(\nabla \times \boldsymbol{h}_{i}, \nabla \times \boldsymbol{h}_{i}-\nabla \times \boldsymbol{h}_{i-1}\right)=\left\|\nabla \times \boldsymbol{h}_{j}\right\|^{2}-\left\|\nabla \times \boldsymbol{H}_{0}\right\|^{2}+\sum_{i=1}^{j}\left\|\nabla \times \boldsymbol{h}_{i}-\nabla \times \boldsymbol{h}_{i-1}\right\|^{2}
$$

and

$$
\sum_{i=1}^{j}\left(\mathcal{K}_{0} \star \boldsymbol{h}_{i}, \nabla \times \delta \boldsymbol{h}_{i}\right) \tau=\left(\mathcal{K}_{0} \star \boldsymbol{h}_{j}, \nabla \times \boldsymbol{h}_{j}\right)-\left(\mathcal{K}_{0} \star \boldsymbol{h}_{0}, \nabla \times \boldsymbol{h}_{0}\right)-\sum_{i=1}^{j}\left(\mathcal{K}_{0} \star \delta \boldsymbol{h}_{i}, \nabla \times \boldsymbol{h}_{i-1}\right) \tau .
$$

Hence, using $(i)$, we obtain

$$
\left|\sum_{i=1}^{j}\left(\mathcal{K}_{0} \star \boldsymbol{h}_{i}, \nabla \times \delta \boldsymbol{h}_{i}\right) \tau\right| \leqslant C_{\varepsilon}+\varepsilon\left\|\nabla \times \boldsymbol{h}_{j}\right\|^{2}+\varepsilon \sum_{i=1}^{j}\left\|\delta \boldsymbol{h}_{i}\right\|^{2} \tau .
$$

The RHS can be estimated using the Cauchy and Young inequalities as follows

$$
\sum_{i=1}^{j}\left(\boldsymbol{f}_{i}, \delta \boldsymbol{h}_{i}\right) \tau \leqslant \varepsilon \sum_{i=1}^{j}\left\|\delta \boldsymbol{h}_{i}\right\|^{2} \tau+C_{\varepsilon} \sum_{i=1}^{j}\left\|\boldsymbol{f}_{i}\right\|^{2} \tau \leqslant C_{\varepsilon}+\varepsilon \sum_{i=1}^{j}\left\|\delta \boldsymbol{h}_{i}\right\|^{2} \tau .
$$

Putting things together and fixing a sufficiently small positive $\varepsilon$, we conclude the proof.

(iv) We set

$$
\delta \boldsymbol{h}_{0}:=\boldsymbol{F}(0)-\nabla \times \nabla \times \boldsymbol{H}_{0}-\nabla \times\left(\mathcal{K}_{0} \star \boldsymbol{H}_{0}\right) .
$$

We subtract (4.1) for $i=i-1$ from (4.1), then we set $\boldsymbol{\varphi}=\delta \boldsymbol{h}_{i}$ and we sum the result for $i=1, \ldots, j$ with $1 \leqslant j \leqslant n$ to get

$$
\sum_{i=1}^{j}\left(\delta^{2} \boldsymbol{h}_{i}, \delta \boldsymbol{h}_{i}\right) \tau+\sum_{i=1}^{j}\left\|\nabla \times \delta \boldsymbol{h}_{i}\right\|^{2} \tau+\sum_{i=1}^{j}\left(\mathcal{K}_{0} \star \delta \boldsymbol{h}_{i}, \nabla \times \delta \boldsymbol{h}_{i}\right) \tau=\sum_{i=1}^{j}\left(\delta \boldsymbol{f}_{i}, \delta \boldsymbol{h}_{i}\right) \tau .
$$

Further, we follow the same way as in $(i)$ when considering $\delta \boldsymbol{h}_{i}$ instead of $\boldsymbol{h}_{i}$.

The existence of a weak solution is proved using Rothe's method. The following piecewise linear in time vector fields $\boldsymbol{H}_{n}$

$$
\begin{aligned}
\boldsymbol{H}_{n}(0) & =\boldsymbol{H}_{0} \\
\boldsymbol{H}_{n}(t) & =\boldsymbol{h}_{i-1}+\left(t-t_{i-1}\right) \delta \boldsymbol{h}_{i} \quad \text { for } t \in\left(t_{i-1}, t_{i}\right], \quad i=1, \ldots, n
\end{aligned}
$$

and the piecewise constant in time fields $\overline{\boldsymbol{H}}_{n}$ are introduced

$$
\overline{\boldsymbol{H}}_{n}(0)=\boldsymbol{H}_{0}, \quad \overline{\boldsymbol{H}}_{n}(t)=\boldsymbol{h}_{i}, \quad \text { for } t \in\left(t_{i-1}, t_{i}\right], \quad i=1, \ldots, n .
$$

Similary, the vector field $\overline{\boldsymbol{F}}_{n}$ is defined. The variational formulation (4.1) can be rewritten as

$$
\left(\partial_{t} \boldsymbol{H}_{n}(t), \boldsymbol{\varphi}\right)+\left(\nabla \times \overline{\boldsymbol{H}}_{n}(t), \nabla \times \boldsymbol{\varphi}\right)+\left(\mathcal{K}_{0} \star \overline{\boldsymbol{H}}_{n}(t), \nabla \times \boldsymbol{\varphi}\right)=\left(\overline{\boldsymbol{F}}_{n}(t), \boldsymbol{\varphi}\right) .
$$

Now, the convergence of the sequences $\boldsymbol{H}_{n}$ and $\overline{\boldsymbol{H}}_{n}$ to the unique weak solution of (3.1) is proved if $\tau \rightarrow 0$ or $n \rightarrow \infty$. 
Theorem 3 (Existence). Let $\boldsymbol{H}_{0} \in \mathbf{L}^{2}(\Omega)$ and $\boldsymbol{F} \in L^{2}\left((0, T), \mathbf{L}^{2}(\Omega)\right)$. Assume that $\nabla \cdot \boldsymbol{H}_{0}=$ $0=\nabla \cdot \boldsymbol{F}(t)$ for any time $t \in[0, T]$. Then there exists a solution $\boldsymbol{H} \in C\left([0, T], \mathbf{L}^{2}(\Omega)\right) \cap$ $L^{2}\left((0, T), \mathbf{H}_{0}(\mathbf{c u r l}, \Omega)\right)$ with $\partial_{t} \boldsymbol{H} \in L^{2}\left((0, T), \mathbf{H}_{0}^{-1}(\mathbf{c u r l}, \Omega)\right)$, which solves (3.3).

Proof: Let us integrate (4.2) in time to get (for any $t \in(0, T)$ )

$$
\left(\boldsymbol{H}_{n}(t)-\boldsymbol{H}_{0}, \boldsymbol{\varphi}\right)+\int_{0}^{t}\left(\nabla \times \overline{\boldsymbol{H}}_{n}, \nabla \times \boldsymbol{\varphi}\right)+\int_{0}^{t}\left(\mathcal{K}_{0} \star \overline{\boldsymbol{H}}_{n}, \nabla \times \boldsymbol{\varphi}\right)=\int_{0}^{t}\left(\overline{\boldsymbol{F}}_{n}, \boldsymbol{\varphi}\right) .
$$

Clearly $\overline{\boldsymbol{F}}_{n} \rightarrow \boldsymbol{F}$ in $L^{2}\left([0, T], \mathbf{L}^{2}(\Omega)\right)$. Both terms $\int_{0}^{t}\left(\nabla \times \overline{\boldsymbol{H}}_{n}, \nabla \times \boldsymbol{\varphi}\right)$ and $\int_{0}^{t}\left(\mathcal{K}_{0} \star \overline{\boldsymbol{H}}_{n}, \nabla \times \boldsymbol{\varphi}\right)$ are linear bounded functionals in the space $L^{2}\left((0, T), \mathbf{H}_{0}(\mathbf{c u r l}, \Omega)\right)$. Therefore, due to the reflexivity of this space, we may write that $\overline{\boldsymbol{H}}_{n} \rightarrow \boldsymbol{H}$ in $L^{2}\left((0, T), \mathbf{H}_{0}(\mathbf{c u r l}, \Omega)\right)$. An easy calculation gives

$$
\left\|\boldsymbol{H}_{n}(t)-\overline{\boldsymbol{H}}_{n}(t)\right\| \leqslant\left\|\boldsymbol{h}_{i}-\boldsymbol{h}_{i-1}\right\| \quad \text { for } t \in\left[t_{i-1}, t_{i}\right]
$$

and

$$
\lim _{n \rightarrow \infty}\left\|\boldsymbol{H}_{n}(t)-\overline{\boldsymbol{H}}_{n}(t)\right\|=0, \quad t \in[0, T] .
$$

Using [31, Lemma 10], we see that

$$
\mathbf{H}^{\frac{1}{2}}(\Omega) \hookrightarrow \hookrightarrow \mathbf{L}^{2}(\Omega) \cong \mathbf{L}^{2}(\Omega)^{*} \hookrightarrow \mathbf{H}_{0}^{-1}(\operatorname{curl}, \Omega)
$$

Lemma $1(i)$ and $(i i)$ give

$$
\boldsymbol{H}_{n} \in L^{2}\left((0, T), \mathbf{H}_{0}(\mathbf{c u r l}, \Omega)\right), \quad \max _{t \in[0, T]}\left\|\boldsymbol{H}_{n}(t)\right\| \leqslant C, \quad \nabla \cdot \boldsymbol{H}_{n}(t)=0, \quad \forall t \in[0, T]
$$

and reviewing [22, Theorem 3.47], we see that $\boldsymbol{H}_{n} \in L^{2}\left((0, T), \mathbf{H}^{\frac{1}{2}}(\Omega)\right)$. Taking into account the fact that $\partial_{t} \boldsymbol{H}_{n} \in L^{2}\left((0, T), \mathbf{H}_{0}^{-1}(\mathbf{c u r l}, \Omega)\right)$ and using the generalized Aubin-Lions lemma [32, Lemma 7.7], we get that $\left\{\boldsymbol{H}_{n}\right\}$ is compact in the space $L_{2}\left((0, T), \mathbf{L}^{2}(\Omega)\right)$. Therefore, there exists a subsequence of $\boldsymbol{H}_{n}$ (denoted by the same symbol again) for which we have (cf. [33, p. 88])

$$
\boldsymbol{H}_{n}(\boldsymbol{x}, t) \rightarrow \boldsymbol{H}(\boldsymbol{x}, t) \quad \text { for a.e. }(\boldsymbol{x}, t) \in Q_{T} .
$$

Moreover, $\boldsymbol{H}_{n} \rightarrow \boldsymbol{H}$ in $L^{2}\left((0, T), \mathbf{H}^{\frac{1}{2}}(\Omega)\right)$.

Now, we can pass to the limit for $n \rightarrow \infty$ in (4.3) as follows

$$
\begin{array}{cccc}
\left(\boldsymbol{H}_{n}(t)-\boldsymbol{H}_{0}, \boldsymbol{\varphi}\right) & +\int_{0}^{t}\left(\nabla \times \overline{\boldsymbol{H}}_{n}, \nabla \times \boldsymbol{\varphi}\right) & +\int_{0}^{t}\left(\mathcal{K}_{0} \star \overline{\boldsymbol{H}}_{n}, \nabla \times \boldsymbol{\varphi}\right) & =\int_{0}^{t}\left(\overline{\boldsymbol{F}}_{n}, \boldsymbol{\varphi}\right) \\
\downarrow & \downarrow & \downarrow & \downarrow \\
\left(\boldsymbol{H}(t)-\boldsymbol{H}_{0}, \boldsymbol{\varphi}\right) & +\int_{0}^{t}(\nabla \times \boldsymbol{H}, \nabla \times \boldsymbol{\varphi}) & +\int_{0}^{t}\left(\mathcal{K}_{0} \star \boldsymbol{H}, \nabla \times \boldsymbol{\varphi}\right) & =\int_{0}^{t}(\boldsymbol{F}, \boldsymbol{\varphi}) .
\end{array}
$$

This is valid for almost all $t \in(0, T)$. Since all terms containing the time integral are continuous in time, we see that also $\left(\boldsymbol{H}(t)-\boldsymbol{H}_{0}, \boldsymbol{\varphi}\right)$ is continuous in time. Hence, (4.4) holds for all $t \in[0, T]$. Using the stability result $\partial_{t} \boldsymbol{H}_{n} \in L^{2}\left((0, T), \mathbf{H}_{0}^{-1}(\operatorname{curl}, \Omega)\right)$, we have

$$
\begin{array}{cc}
\left(\boldsymbol{H}_{n}(t)-\boldsymbol{H}_{0}, \boldsymbol{\varphi}\right) & =\int_{0}^{t}\left(\partial_{t} \boldsymbol{H}_{n}, \boldsymbol{\varphi}\right) \\
\downarrow & \downarrow \\
\left(\boldsymbol{H}(t)-\boldsymbol{H}_{0}, \boldsymbol{\varphi}\right) & =\int_{0}^{t}\left(\partial_{t} \boldsymbol{H}, \boldsymbol{\varphi}\right)
\end{array}
$$


with $\partial_{t} \boldsymbol{H} \in L^{2}\left((0, T), \mathbf{H}_{0}^{-1}(\mathbf{c u r l}, \Omega)\right)$. Differentiating the result of (4.4) with respect to the time variable, we get the existence of a solution to (3.3).

Consider the following evolution triple (or sometimes called Gelfand's triple) of spaces

$$
\mathbf{H}_{0}(\operatorname{curl}, \Omega) \hookrightarrow \mathbf{L}^{2}(\Omega) \cong \mathbf{L}^{2}(\Omega)^{*} \hookrightarrow \mathbf{H}_{0}^{-1}(\operatorname{curl}, \Omega)
$$

We know that

$$
\boldsymbol{H} \in L^{2}\left((0, T), \mathbf{H}_{0}(\operatorname{curl}, \Omega)\right) \quad \text { and } \quad \partial_{t} \boldsymbol{H} \in L^{2}\left((0, T), \mathbf{H}_{0}^{-1}(\operatorname{curl}, \Omega)\right) .
$$

Applying [32, Lemma 7.3], we get $\boldsymbol{H} \in C\left([0, T], \mathbf{L}^{2}(\Omega)\right)$, which concludes the proof.

The following theorem addresses the error estimates for the time discretization.

Theorem 4 (Error). Suppose that $\boldsymbol{F} \in \operatorname{Lip}\left([0, T], \mathbf{L}^{2}(\Omega)\right)$.

(i) If $\boldsymbol{H}_{0} \in \mathbf{H}_{0}(\operatorname{curl}, \Omega)$ then

$$
\max _{t \in[0, T]}\left\|\boldsymbol{H}_{n}(t)-\boldsymbol{H}(t)\right\|^{2}+\int_{0}^{T}\left\|\nabla \times\left[\boldsymbol{H}_{n}-\boldsymbol{H}\right]\right\|^{2} \leqslant C \tau .
$$

(ii) If $\nabla \times\left(\mathcal{K}_{0} \star \boldsymbol{H}_{0}\right) \in \mathbf{L}^{2}(\Omega), \boldsymbol{H}_{0} \in \mathbf{H}_{0}(\mathbf{c u r l}, \Omega)$ and $\nabla \times \nabla \times \boldsymbol{H}_{0} \in \mathbf{L}^{2}(\Omega)$ then

$$
\max _{t \in[0, T]}\left\|\boldsymbol{H}_{n}(t)-\boldsymbol{H}(t)\right\|^{2}+\int_{0}^{T}\left\|\nabla \times\left[\boldsymbol{H}_{n}-\boldsymbol{H}\right]\right\|^{2} \leqslant C \tau^{2} .
$$

Please note that the positive constant $C$ in this estimates is of the form $C e^{C T}$.

Proof: We subtract (3.3) from (4.2), set $\boldsymbol{\varphi}=\boldsymbol{H}_{n}-\boldsymbol{H}$ and integrate in time to get

$$
\begin{gathered}
\frac{1}{2}\left\|\boldsymbol{H}_{n}(t)-\boldsymbol{H}(t)\right\|^{2}+\int_{0}^{t}\left\|\nabla \times \boldsymbol{H}_{n}-\nabla \times \boldsymbol{H}\right\|^{2}+\int_{0}^{t}\left(\mathcal{K}_{0} \star\left[\boldsymbol{H}_{n}-\boldsymbol{H}\right], \nabla \times\left[\boldsymbol{H}_{n}-\boldsymbol{H}\right]\right) \\
=\int_{0}^{t}\left(\overline{\boldsymbol{F}}_{n}-\boldsymbol{F}, \boldsymbol{H}_{n}-\boldsymbol{H}\right)+\int_{0}^{t}\left(\nabla \times\left[\boldsymbol{H}_{n}-\overline{\boldsymbol{H}}_{n}\right], \nabla \times\left[\boldsymbol{H}_{n}-\boldsymbol{H}\right]\right) \\
+\int_{0}^{t}\left(\mathcal{K}_{0} \star\left[\boldsymbol{H}_{n}-\overline{\boldsymbol{H}}_{n}\right], \nabla \times\left[\boldsymbol{H}_{n}-\boldsymbol{H}\right]\right) .
\end{gathered}
$$

We may write due to the Lipschitz continuity of $\boldsymbol{F}$ that

$$
\left|\int_{0}^{t}\left(\overline{\boldsymbol{F}}_{n}-\boldsymbol{F}, \boldsymbol{H}_{n}-\boldsymbol{H}\right)\right| \lesssim \int_{0}^{t}\left\|\overline{\boldsymbol{F}}_{n}-\boldsymbol{F}\right\|^{2}+\int_{0}^{t}\left\|\boldsymbol{H}_{n}-\boldsymbol{H}\right\|^{2} \leqslant C \tau^{2}+\int_{0}^{t}\left\|\boldsymbol{H}_{n}-\boldsymbol{H}\right\|^{2} .
$$

It holds

$$
\left\|\boldsymbol{H}_{n}(t)-\overline{\boldsymbol{H}}_{n}(t)\right\| \leqslant \tau\left\|\partial_{t} \boldsymbol{H}_{n}(t)\right\| \quad \text { for } t \in[0, T] .
$$

For the last term of (4.5), we may write using (3.2) and Lemma 1(iii) that

$$
\begin{aligned}
\left|\int_{0}^{t}\left(\mathcal{K}_{0} \star\left[\boldsymbol{H}_{n}-\overline{\boldsymbol{H}}_{n}\right], \nabla \times\left[\boldsymbol{H}_{n}-\boldsymbol{H}\right]\right)\right| & \lesssim \int_{0}^{t}\left\|\boldsymbol{H}_{n}-\overline{\boldsymbol{H}}_{n}\right\|\left\|\nabla \times\left[\boldsymbol{H}_{n}-\boldsymbol{H}\right]\right\| \\
& \leqslant \varepsilon \int_{0}^{t}\left\|\nabla \times\left[\boldsymbol{H}_{n}-\boldsymbol{H}\right]\right\|^{2}+C_{\varepsilon} \int_{0}^{t}\left\|\boldsymbol{H}_{n}-\overline{\boldsymbol{H}}_{n}\right\|^{2} \\
& \leqslant \varepsilon \int_{0}^{t}\left\|\nabla \times\left[\boldsymbol{H}_{n}-\boldsymbol{H}\right]\right\|^{2}+C_{\varepsilon} \tau^{2} .
\end{aligned}
$$


Analogously, we have that

$$
\left|\int_{0}^{t}\left(\mathcal{K}_{0} \star\left[\boldsymbol{H}_{n}-\boldsymbol{H}\right], \nabla \times\left[\boldsymbol{H}_{n}-\boldsymbol{H}\right]\right)\right| \leqslant \varepsilon \int_{0}^{t}\left\|\nabla \times\left[\boldsymbol{H}_{n}-\boldsymbol{H}\right]\right\|^{2}+C_{\varepsilon} \int_{0}^{t}\left\|\boldsymbol{H}_{n}-\boldsymbol{H}\right\|^{2} .
$$

It remains to estimate the second term on the RHS in (4.5). Here, we have to distinguish between two cases depending on the a priori estimates we have (see Lemma 1(iii) and (iv)):

$$
\begin{aligned}
\left|\int_{0}^{t}\left(\nabla \times\left[\boldsymbol{H}_{n}-\overline{\boldsymbol{H}}_{n}\right], \nabla \times\left[\boldsymbol{H}_{n}-\boldsymbol{H}\right]\right)\right| & \leqslant \varepsilon \int_{0}^{t}\left\|\nabla \times\left[\boldsymbol{H}_{n}-\boldsymbol{H}\right]\right\|^{2}+C_{\varepsilon} \int_{0}^{t}\left\|\nabla \times\left[\boldsymbol{H}_{n}-\overline{\boldsymbol{H}}_{n}\right]\right\|^{2} \\
& \leqslant \varepsilon \int_{0}^{t}\left\|\nabla \times\left[\boldsymbol{H}_{n}-\boldsymbol{H}\right]\right\|^{2}+C_{\varepsilon} \tau, \\
\left|\int_{0}^{t}\left(\nabla \times\left[\boldsymbol{H}_{n}-\overline{\boldsymbol{H}}_{n}\right], \nabla \times\left[\boldsymbol{H}_{n}-\boldsymbol{H}\right]\right)\right| & \leqslant \varepsilon \int_{0}^{t}\left\|\nabla \times\left[\boldsymbol{H}_{n}-\boldsymbol{H}\right]\right\|^{2}+C_{\varepsilon} \int_{0}^{t}\left\|\nabla \times\left[\boldsymbol{H}_{n}-\overline{\boldsymbol{H}}_{n}\right]\right\|^{2} \\
& \leqslant \varepsilon \int_{0}^{t}\left\|\nabla \times\left[\boldsymbol{H}_{n}-\boldsymbol{H}\right]\right\|^{2}+C_{\varepsilon} \tau^{2} .
\end{aligned}
$$

Putting things together, choosing a sufficiently small positive $\varepsilon$ and applying Grönwall's argument, we conclude the proof.

\section{Modified scheme}

In this section, the following time-discrete scheme is considered, which represents a slight modification of (4.1)

$$
\left\{\begin{aligned}
\left(\delta \boldsymbol{h}_{i}, \boldsymbol{\varphi}\right)+\left(\nabla \times \boldsymbol{h}_{i}, \nabla \times \boldsymbol{\varphi}\right) & =\left(\boldsymbol{f}_{i}, \boldsymbol{\varphi}\right)-\left(\mathcal{K}_{0} \star \boldsymbol{h}_{i-1}, \nabla \times \boldsymbol{\varphi}\right), \quad \boldsymbol{\varphi} \in \mathbf{H}_{0}(\operatorname{curl}, \Omega) ; \\
\boldsymbol{h}_{0} & =\boldsymbol{H}_{0}
\end{aligned}\right.
$$

which is equivalent to

$$
\begin{aligned}
a\left(\boldsymbol{h}_{i}, \boldsymbol{\varphi}\right) & :=\left(\frac{\boldsymbol{h}_{i}}{\tau}, \boldsymbol{\varphi}\right)+\left(\nabla \times \boldsymbol{h}_{i}, \nabla \times \boldsymbol{\varphi}\right) \\
& =\left(\boldsymbol{f}_{i}, \boldsymbol{\varphi}\right)-\left(\mathcal{K}_{0} \star \boldsymbol{h}_{i-1}, \nabla \times \boldsymbol{\varphi}\right)+\left(\frac{\boldsymbol{h}_{i-1}}{\tau}, \boldsymbol{\varphi}\right)=: f_{i}(\boldsymbol{\varphi}) .
\end{aligned}
$$

Here the convolution term is taken explicitly (from the last time step), while (4.1) considers an implicit form (from the actual time step). Therefore, this scheme is semi-implicit.

The bilinear form $a(\boldsymbol{h}, \boldsymbol{\varphi})$ is elliptic and continuous in $\mathbf{H}_{0}(\mathbf{c u r l}, \Omega)$. According to (3.2), the functional $f_{i}(\boldsymbol{\varphi})$ is linear and bounded in $\mathbf{H}_{0}(\mathbf{c u r l}, \Omega)$ if $\boldsymbol{H}_{i-1} \in \mathbf{L}^{2}(\Omega)$. Thus, if $\boldsymbol{H}_{0} \in \mathbf{L}^{2}(\Omega)$, an application of the Lax-Milgram lemma gives the existence of a unique solution to (5.1) for any $i=1, \ldots, n$ and any $\tau>0$.

Handling this scheme is very similar to the way used for (4.1). For short, only the differences between both algorithms are pointed out.

Lemma 2 (A priori estimates). Suppose that $\boldsymbol{F} \in L^{2}\left((0, T), \mathbf{L}^{2}(\Omega)\right)$.

(i) Let $\boldsymbol{H}_{0} \in \mathbf{L}^{2}(\Omega)$. Then, there exists a positive constant $C$ such that

$$
\max _{1 \leqslant i \leqslant n}\left\|\boldsymbol{h}_{i}\right\|^{2}+\sum_{i=1}^{n}\left\|\boldsymbol{h}_{i}-\boldsymbol{h}_{i-1}\right\|^{2}+\sum_{i=1}^{n}\left\|\nabla \times \boldsymbol{h}_{i}\right\|^{2} \tau \leqslant C .
$$

(ii) If $\nabla \cdot \boldsymbol{H}_{0}=0=\nabla \cdot \boldsymbol{F}$ then $\nabla \cdot \boldsymbol{h}_{i}=0$ for all $i=1, \ldots, n$. Furthermore, we have that

$$
\tau \sum_{i=1}^{n}\left\|\delta \boldsymbol{h}_{i}\right\|_{\mathbf{H}_{0}^{-1}(\operatorname{curl}, \Omega)}^{2} \leqslant C .
$$


(iii) If $\nabla \times\left(\mathcal{K}_{0} \star \boldsymbol{H}_{0}\right) \in \mathbf{L}^{2}(\Omega), \boldsymbol{H}_{0} \in \mathbf{H}_{0}(\mathbf{c u r l}, \Omega)$ and $\nabla \times \nabla \times \boldsymbol{H}_{0} \in \mathbf{L}^{2}(\Omega)$ then

$$
\max _{1 \leqslant i \leqslant n}\left\|\nabla \times \boldsymbol{h}_{i}\right\|^{2}+\sum_{i=1}^{n}\left\|\nabla \times \boldsymbol{h}_{i}-\nabla \times \boldsymbol{h}_{i-1}\right\|^{2}+\sum_{i=1}^{n}\left\|\delta \boldsymbol{h}_{i}\right\|^{2} \tau \leqslant C .
$$

(iv) If $\boldsymbol{F}(0) \in \mathbf{L}^{2}(\Omega), \partial_{t} \boldsymbol{F} \in L^{2}\left((0, T), \mathbf{L}^{2}(\Omega)\right), \nabla \times\left(\mathcal{K}_{0} \star \boldsymbol{H}_{0}\right) \in \mathbf{L}^{2}(\Omega), \boldsymbol{H}_{0} \in \mathbf{H}_{0}(\mathbf{c u r l}, \Omega)$ and $\nabla \times \nabla \times \boldsymbol{H}_{0} \in \mathbf{L}^{2}(\Omega)$ then

$$
\max _{1 \leqslant i \leqslant n}\left\|\delta \boldsymbol{h}_{i}\right\|^{2}+\sum_{i=1}^{n}\left\|\delta \boldsymbol{h}_{i}-\delta \boldsymbol{h}_{i-1}\right\|^{2}+\sum_{i=1}^{n}\left\|\nabla \times \delta \boldsymbol{h}_{i}\right\|^{2} \tau \leqslant C .
$$

Proof: $(i)$ We follow Lemma 1(i). Using (3.2) we have

$$
\left|\sum_{i=1}^{j}\left(\mathcal{K}_{0} \star \boldsymbol{h}_{i-1}, \nabla \times \boldsymbol{h}_{i}\right) \tau\right| \leqslant \varepsilon \sum_{i=1}^{j}\left\|\nabla \times \boldsymbol{h}_{i}\right\|^{2} \tau+C_{\varepsilon} \sum_{i=0}^{j}\left\|\boldsymbol{h}_{i}\right\|^{2} \tau .
$$

After fixing a sufficiently small positive $\varepsilon$, an application of Grönwall's lemma completes the proof.

(ii) The proof is the same as in Lemma $1(i i)$ replacing $\left(\mathcal{K}_{0} \star \boldsymbol{h}_{i}, \nabla \times \boldsymbol{\varphi}\right)$ by $\left(\mathcal{K}_{0} \star \boldsymbol{h}_{i-1}, \nabla \times \boldsymbol{\varphi}\right)$.

(iii) We set

$$
\delta \boldsymbol{h}_{0}:=\boldsymbol{F}(0)-\nabla \times \nabla \times \boldsymbol{H}_{0}-\nabla \times\left(\mathcal{K}_{0} \star \boldsymbol{H}_{0}\right), \quad \boldsymbol{h}_{-1}:=\boldsymbol{h}_{0}-\delta \boldsymbol{h}_{0} \tau .
$$

Please note that $\delta \boldsymbol{h}_{0}, \boldsymbol{h}_{-1} \in \mathbf{L}^{2}(\Omega)$. The verification is the same as in Lemma 1(iii) replacing $\left(\mathcal{K}_{0} \star \boldsymbol{h}_{i}, \nabla \times \varphi\right)$ by $\left(\mathcal{K}_{0} \star \boldsymbol{h}_{i-1}, \nabla \times \varphi\right)$. Remark that

$$
\sum_{i=1}^{j}\left(\mathcal{K}_{0} \star \boldsymbol{h}_{i-1}, \nabla \times \delta \boldsymbol{h}_{i}\right) \tau=\left(\mathcal{K}_{0} \star \boldsymbol{h}_{j}, \nabla \times \boldsymbol{h}_{j}\right)-\left(\mathcal{K}_{0} \star \boldsymbol{h}_{0}, \nabla \times \boldsymbol{h}_{0}\right)-\sum_{i=1}^{j}\left(\mathcal{K}_{0} \star \delta \boldsymbol{h}_{i}, \nabla \times \boldsymbol{h}_{i}\right) \tau .
$$

(iv) The proof follows very closely the proof of Lemma $1(i v)$, except for the appearance of the term $\left(\mathcal{K}_{0} \star \boldsymbol{h}_{i-1}, \nabla \times \boldsymbol{\varphi}\right)$ instead of $\left(\mathcal{K}_{0} \star \boldsymbol{h}_{i}, \nabla \times \boldsymbol{\varphi}\right)$.

The variational formulation (5.1) can be rewritten as

$$
\left(\partial_{t} \boldsymbol{H}_{n}(t), \boldsymbol{\varphi}\right)+\left(\nabla \times \overline{\boldsymbol{H}}_{n}(t), \nabla \times \boldsymbol{\varphi}\right)=\left(\overline{\boldsymbol{F}}_{n}(t), \boldsymbol{\varphi}\right)-\left(\mathcal{K}_{0} \star \overline{\boldsymbol{H}}_{n}(t-\tau), \nabla \times \boldsymbol{\varphi}\right) .
$$

Next theorem derives the error estimates for the scheme (5.1).

Theorem 5 (Error). Suppose that $\boldsymbol{F} \in \operatorname{Lip}\left([0, T], \mathbf{L}^{2}(\Omega)\right)$.

(i) If $\boldsymbol{H}_{0} \in \mathbf{H}_{0}(\operatorname{curl}, \Omega)$ then

$$
\max _{t \in[0, T]}\left\|\boldsymbol{H}_{n}(t)-\boldsymbol{H}(t)\right\|^{2}+\int_{0}^{T}\left\|\nabla \times\left[\boldsymbol{H}_{n}-\boldsymbol{H}\right]\right\|^{2} \leqslant C \tau .
$$

(ii) If $\nabla \times\left(\mathcal{K}_{0} \star \boldsymbol{H}_{0}\right) \in \mathbf{L}^{2}(\Omega), \boldsymbol{H}_{0} \in \mathbf{H}_{0}(\mathbf{c u r l}, \Omega)$ and $\nabla \times \nabla \times \boldsymbol{H}_{0} \in \mathbf{L}^{2}(\Omega)$ then

$$
\max _{t \in[0, T]}\left\|\boldsymbol{H}_{n}(t)-\boldsymbol{H}(t)\right\|^{2}+\int_{0}^{T}\left\|\nabla \times\left[\boldsymbol{H}_{n}-\boldsymbol{H}\right]\right\|^{2} \leqslant C \tau^{2} .
$$

Please note that the positive constant $C$ in this estimates is of the form $C e^{C T}$.

Proof: We follow Theorem 4. We get (4.5), where the term $\int_{0}^{t}\left(\mathcal{K}_{0} \star\left[\boldsymbol{H}_{n}-\overline{\boldsymbol{H}}_{n}\right], \nabla \times\left[\boldsymbol{H}_{n}-\boldsymbol{H}\right]\right)$ 
is replaced by $\int_{0}^{t}\left(\mathcal{K}_{0} \star\left[\boldsymbol{H}_{n}(s)-\overline{\boldsymbol{H}}_{n}(s-\tau)\right], \nabla \times\left[\boldsymbol{H}_{n}(s)-\boldsymbol{H}(s)\right]\right) \mathrm{d} s$. This can be handled using (3.2) and Lemma $2($ iii) as follows

$$
\begin{aligned}
& \left|\int_{0}^{t}\left(\mathcal{K}_{0} \star\left[\boldsymbol{H}_{n}(s)-\overline{\boldsymbol{H}}_{n}(s-\tau)\right], \nabla \times\left[\boldsymbol{H}_{n}(s)-\boldsymbol{H}(s)\right]\right) \mathrm{d} s\right| \\
& \quad \lesssim \int_{0}^{t}\left\|\boldsymbol{H}_{n}(s)-\overline{\boldsymbol{H}}_{n}(s-\tau)\right\|\left\|\nabla \times\left[\boldsymbol{H}_{n}(s)-\boldsymbol{H}(s)\right]\right\| \mathrm{d} s \\
& \quad \leqslant \varepsilon \int_{0}^{t}\left\|\nabla \times\left[\boldsymbol{H}_{n}(s)-\boldsymbol{H}(s)\right]\right\|^{2} \mathrm{~d} s+C_{\varepsilon} \int_{0}^{t}\left\|\boldsymbol{H}_{n}(s)-\overline{\boldsymbol{H}}_{n}(s-\tau)\right\|^{2} \mathrm{~d} s \\
& \quad \leqslant \varepsilon \int_{0}^{t}\left\|\nabla \times\left[\boldsymbol{H}_{n}(s)-\boldsymbol{H}(s)\right]\right\|^{2} \mathrm{~d} s+C_{\varepsilon} \tau^{2} .
\end{aligned}
$$

The rest is the same as in Theorem 4 .

This second scheme is considered, because it is easier to implement than the first scheme and it gives the same order of convergence. Moreover, the finite element matrix corresponding with the LHS of (6.9) is less dense and hence less memory is needed.

\section{Higher regularity}

The problem (3.1) is non-symmetric, due to the convolution term. It means that the term $\left(\mathcal{K}_{0} \star \boldsymbol{H}, \nabla \times \boldsymbol{H}\right)$ in the a priori estimates exists of two terms with derivatives of a different order. The unique solution of problem (3.1) can be approached by the scheme (4.1) or (5.1). Theorems 4 and 5 claim to have optimal convergence rates $\mathcal{O}(\tau)$ in the space $C\left([0, T], \mathbf{L}^{2}(\Omega)\right) \cap L_{2}((0, T), \mathbf{H}(\mathbf{c u r l}, \Omega))$ under appropriate conditions. This error estimate has been obtained using a priori estimates, which were based on Grönwall's argument. Therefore, $\mathcal{O}(\tau)=e^{C T} \tau$, which means that the constant $e^{C T}$ may be large. To get rid of the exponential (in time) character of this constant, the use of Grönwall's lemma should be avoided. This can be done by symmetrification of the problem, namely by incorporation of the curl operator $\nabla \times \boldsymbol{J}_{s}$ into a new convolution kernel, cf. [13] and [19, §11.7]. The term $\left(\mathcal{K}_{0} \star \boldsymbol{H}, \nabla \times \boldsymbol{H}\right)$ will be replaced by $(\mathcal{K} \star \boldsymbol{H}, \boldsymbol{H})$, where $\mathcal{K}$ is defined in the following lemma.

Lemma 3. Let $\boldsymbol{J}_{s}$ be defined as in (1.4). Suppose that $\nabla \cdot \boldsymbol{H}=0$ in $\Omega$ and $\boldsymbol{H} \cdot \boldsymbol{\nu}=0$ on $\partial \Omega$. Then,

$$
\nabla \times \boldsymbol{J}_{s}(\boldsymbol{x})=-\int_{\Omega} \mathcal{K}\left(\boldsymbol{x}, \boldsymbol{x}^{\prime}\right) \boldsymbol{H}\left(\boldsymbol{x}^{\prime}\right) d \boldsymbol{x}^{\prime}=:-(\mathcal{K} \star \boldsymbol{H})(\boldsymbol{x}), \quad \boldsymbol{x} \in \Omega,
$$

where the kernel $\mathcal{K}$ is defined by

$$
\mathcal{K}: \Omega \times \Omega \rightarrow \mathbb{R}:\left(\boldsymbol{x}, \boldsymbol{x}^{\prime}\right) \mapsto \kappa\left(\left|\boldsymbol{x}-\boldsymbol{x}^{\prime}\right|\right),
$$

with

$$
\kappa:(0, \infty) \rightarrow \mathbb{R}: s \mapsto \begin{cases}\frac{\widetilde{C}}{2 s^{2}}\left(1-\frac{s}{r_{0}}\right) \exp \left(-\frac{s}{r_{0}}\right) & s<r_{0} \\ 0 & s \geqslant r_{0}\end{cases}
$$

Proof: (Adapted from [13]) Let $\boldsymbol{r}=\boldsymbol{x}-\boldsymbol{x}^{\prime}$ and $r=|\boldsymbol{r}|$. The first component is given by

$$
\begin{aligned}
\left(\nabla \times \boldsymbol{J}_{s}\right)_{1}(\boldsymbol{x})=\int_{\Omega}\left[H_{2}\left(\boldsymbol{x}^{\prime}\right) \partial_{x_{2}}\left(\sigma_{0}(r) r_{1}\right)\right. & +H_{3}\left(\boldsymbol{x}^{\prime}\right) \partial_{x_{3}}\left(\sigma_{0}(r) r_{1}\right) \\
& \left.-H_{1}\left(\boldsymbol{x}^{\prime}\right) \partial_{x_{2}}\left(\sigma_{0}(r) r_{2}\right)-H_{1}\left(\boldsymbol{x}^{\prime}\right) \partial_{x_{3}}\left(\sigma_{0}(r) r_{3}\right)\right] \mathrm{d} \boldsymbol{x}^{\prime} .
\end{aligned}
$$

Remark that $\partial_{x_{i}} \sigma_{0}(r)=\sigma_{0}^{\prime}(r) \frac{r_{i}}{r}$ and $\partial_{x_{i}^{\prime}} \sigma_{0}(r)=-\sigma_{0}^{\prime}(r) \frac{r_{i}}{r}$. A simple calculation gives that $\partial_{x_{j}}=$ $-\partial_{x_{j}^{\prime}}$ on the term $\sigma_{0}(r) r_{i}$. We apply this on the first two terms of the integrand together with the 
productrule

$$
\partial_{x_{j}^{\prime}}\left(\sigma_{0}(r) r_{l} H_{m}\left(\boldsymbol{x}^{\prime}\right)\right)=\sigma_{0}(r) r_{l} \partial_{x_{j}^{\prime}} H_{m}\left(\boldsymbol{x}^{\prime}\right)+H_{m}\left(\boldsymbol{x}^{\prime}\right) \partial_{x_{j}^{\prime}}\left(\sigma_{0}(r) r_{l}\right) .
$$

Applying $\nabla \cdot \boldsymbol{H}=0$ on the terms corresponding with the first term of the RHS of the productrule leaves

$$
\begin{array}{r}
\left(\nabla \times \boldsymbol{J}_{s}\right)_{1}(\boldsymbol{x})=\int_{\Omega}\left[-\partial_{x_{2}^{\prime}}\left(\sigma_{0}(r) r_{1} H_{2}\left(\boldsymbol{x}^{\prime}\right)\right)-\partial_{x_{3}^{\prime}}\left(\sigma_{0}(r) r_{1} H_{3}\left(\boldsymbol{x}^{\prime}\right)\right)-\sigma_{0}(r) r_{1} \partial_{x_{1}^{\prime}} H_{1}\left(\boldsymbol{x}^{\prime}\right)\right. \\
\left.-H_{1}\left(\boldsymbol{x}^{\prime}\right) \partial_{x_{2}}\left(\sigma_{0}(r) r_{2}\right)-H_{1}\left(\boldsymbol{x}^{\prime}\right) \partial_{x_{3}}\left(\sigma_{0}(r) r_{3}\right)\right] \mathrm{d} \boldsymbol{x}^{\prime} .
\end{array}
$$

Application of the divergence theorem on the first two terms in the integrand and integration by parts on the third term in the integrand gives that $\left(\nabla \times \boldsymbol{J}_{s}\right)_{1}(\boldsymbol{x})$ equals

$$
\begin{aligned}
\int_{\partial \Omega}\left[-\sigma_{0}(r) r_{1}\right. & \left.H_{2}\left(\boldsymbol{x}^{\prime}\right) \nu_{2}\left(\boldsymbol{x}^{\prime}\right)-\sigma_{0}(r) r_{1} H_{3}\left(\boldsymbol{x}^{\prime}\right) \nu_{3}\left(\boldsymbol{x}^{\prime}\right)-\sigma_{0}(r) r_{1} H_{1}\left(\boldsymbol{x}^{\prime}\right) \nu_{1}\left(\boldsymbol{x}^{\prime}\right)\right] \mathrm{d} \boldsymbol{x}^{\prime} \\
& +\int_{\Omega}\left[H_{1}\left(\boldsymbol{x}^{\prime}\right) \partial_{x_{1}^{\prime}}\left(\sigma_{0}(r) r_{1}\right)-H_{1}\left(\boldsymbol{x}^{\prime}\right) \partial_{x_{2}}\left(\sigma_{0}(r) r_{2}\right)-H_{1}\left(\boldsymbol{x}^{\prime}\right) \partial_{x_{3}}\left(\sigma_{0}(r) r_{3}\right)\right] \mathrm{d} \boldsymbol{x}^{\prime} .
\end{aligned}
$$

Firstly, we apply $\partial_{x_{j}^{\prime}}=-\partial_{x_{j}}$ on the first term in the second integral. Secondly, the surface and volume integrals are combined. Equivalently, we can prove an analogous result for the second and third component of the curl of $\boldsymbol{J}_{s}$. Consequently,

$$
\left(\nabla \times \boldsymbol{J}_{s}\right)_{i}(\boldsymbol{x})=-\int_{\Omega} H_{i}\left(\boldsymbol{x}^{\prime}\right) \nabla \cdot\left(\sigma_{0}(r) \boldsymbol{r}\right) \mathrm{d} \boldsymbol{x}^{\prime}-\int_{\partial \Omega} \sigma_{0}(r) r_{i} \boldsymbol{H}\left(\boldsymbol{x}^{\prime}\right) \cdot \boldsymbol{\nu}\left(\boldsymbol{x}^{\prime}\right) \mathrm{d} \boldsymbol{x}^{\prime},
$$

for $i=1,2,3$. From the assumption $\boldsymbol{H} \cdot \boldsymbol{\nu}=0$ on $\partial \Omega$, we conclude the proof. Therefore,

$$
\kappa(r)=\kappa(|\boldsymbol{r}|)=\nabla \cdot\left(\sigma_{0}(r) \boldsymbol{r}\right)=\partial \boldsymbol{r} \cdot\left(\sigma_{0}(r) \boldsymbol{r}\right)=r \sigma_{0}^{\prime}(r)+3 \sigma_{0}(r) .
$$

A simple calculation using (1.5) gives the exact form of the kernel $\mathcal{K}$.

\subsection{Properties of the kernel $\mathcal{K}$}

The goal of this section is to show that the kernel $\mathcal{K}$, defined in (6.1), is positive definite. This characteristic is useful for simplifying proofs and for avoiding the Grönwall argument. The starting point is a definition of a positive definite kernel in the sense of Mercer (cf. [34]) and a radial function (cf. [35]).

Definition 6.1. Let $X \subset \mathbb{R}^{d}, d \geqslant 1$. A symmetric kernel $\hat{\mathcal{K}}: X \times X \rightarrow \mathbb{R}$ is called positive definite if and only if

$$
\int_{X}\left(\int_{X} \hat{\mathcal{K}}\left(\boldsymbol{x}, \boldsymbol{x}^{\prime}\right) \boldsymbol{f}\left(\boldsymbol{x}^{\prime}\right) d \boldsymbol{x}^{\prime}\right) \boldsymbol{f}(\boldsymbol{x}) d \boldsymbol{x} \geqslant \mathbf{0}, \quad \forall \boldsymbol{f} \in \mathbf{L}_{1}(X) .
$$

Definition 6.2. A function $\Psi: \mathbb{R}^{d} \rightarrow \mathbb{R}, d \geqslant 1$, is called radial provided there exists a univariate function $\varphi:[0, \infty) \rightarrow \mathbb{R}$ such that

$$
\Psi(\boldsymbol{x})=\varphi(r) \text { where } r=|\boldsymbol{x}| .
$$

Remark 1. $\mathcal{K}$ is a radial function with $\varphi=\kappa$ and $r=\left|\boldsymbol{x}-\boldsymbol{x}^{\prime}\right|$, see Theorem 3 .

The following important lemma is easy to show using spherical coordinates, so its proof is omitted.

Lemma 4. $\mathcal{K}(\boldsymbol{x}, \cdot) \in L_{p}(\Omega)$ if $1 \leqslant p<\frac{3}{2}, \forall \boldsymbol{x} \in \Omega$.

The theory of completely monotone functions is involved to prove that $\mathcal{K}$ is positive definite. 
Definition 6.3. A function $\varphi:(0, a) \rightarrow \mathbb{R}$ that is in $C(0, a)$ and satisfies

$$
(-1)^{l} \varphi^{(l)}(x) \geq 0, \quad x>0, \quad l=0,1,2, \ldots,
$$

is called completely monotone on $(0, a)$. The limit $\varphi^{(l)}(0)=\lim _{x \searrow 0} \varphi^{(l)}(x)$, finite or infinite, exists.

Schoenberg has pointed out the following connection between positive definite radial and completely monotone functions (cf. [36, Thm. 3]). A more recent reference is [37].

Theorem 6 (Schoenberg interpolation theorem). A function $\varphi$ is completely monotone on $(0, \infty)$ if and only if $\Psi=\varphi\left(|\cdot|^{2}\right)$ is positive definite and radial on $\mathbb{R}^{d}$ for all $d \geqslant 1$.

Lemma 5. The kernel $\mathcal{K}$, defined in Lemma 3, is positive definite on $\Omega \times \Omega \subset \mathbb{R}^{d} \times \mathbb{R}^{d}$.

Proof: The following functions are completely monotone on $(0, \infty)$ (proof by induction):

- $\hat{\kappa}_{1}(r)=\frac{C}{2}, \quad C \geqslant 0$;

- $\hat{\kappa}_{2}(r)=\frac{1}{r}$, since for $l=0,1,2, \ldots$

$$
(-1)^{l} \hat{\kappa}_{2}^{(l)}(r)=(-1)^{2 l} l ! r^{-(l+1)} \geqslant 0, \quad r>0 ;
$$

- $\hat{\kappa}_{3}(r)=\exp \left(-\frac{\sqrt{r}}{r_{0}}\right) \geq 0$, since for $l=1,2, \ldots$

$$
(-1)^{l} \hat{\kappa}_{3}^{(l)}(r)=(-1)^{2 l} 2^{-l} \exp \left(-\frac{\sqrt{r}}{r_{0}}\right) \sum_{i=0}^{l-1} C_{i}^{(l)} r_{0}^{i-l} r^{-\frac{l+i}{2}} \geqslant 0, \quad r>0,
$$

with

$$
C_{i}^{(l)}= \begin{cases}C_{i}^{(l-1)} & i=0 \\ C_{i}^{(l-1)}+C_{i-1}^{(l-1)}(l+i-2) & 1 \leqslant i \leqslant l-1 \\ C_{i-1}^{(l-1)}(l+i-2) & i=l-1\end{cases}
$$

and

$$
C_{0}^{(1)}=1
$$

- $\hat{\kappa}_{4}(r)=\left\{\begin{array}{ll}1-\frac{\sqrt{r}}{r_{0}} & r<r_{0}^{2} ; \\ 0 & r \geqslant r_{0}^{2} ;\end{array}\right.$ since for $l=1,2, \ldots$

$$
(-1)^{l} \hat{\kappa}_{4}^{(l)}(r)= \begin{cases}(-1)^{2 l} \frac{\Gamma\left(l-\frac{1}{2}\right)}{2 \sqrt{\pi} r_{0} r^{l-\frac{1}{2}}} \geq 0 & 0<r<r_{0}^{2} \\ 0 & r \geqslant r_{0}^{2}\end{cases}
$$

where $\Gamma$ denotes the gamma function.

From the previous calculations follows that the function $\hat{\kappa}(r)=\hat{\kappa}_{1}(r) \hat{\kappa}_{2}(r) \hat{\kappa}_{3}(r) \hat{\kappa}_{4}(r)$ is completely monotone on $(0, \infty)$ (the product of two completely monotone functions is completely monotone, $\mathrm{cf}$. [38]). The Schoenberg interpolation Theorem 6 tells us that $\mathcal{K}\left(\boldsymbol{x}, \boldsymbol{x}^{\prime}\right)=\hat{\kappa}\left(\left|\boldsymbol{x}-\boldsymbol{x}^{\prime}\right|^{2}\right)$ is positive definite and radial on $\mathbb{R}^{d} \times \mathbb{R}^{d}$ for all $d$.

\subsection{Symmetric problem}

The solution of problem (3.1) is divergence free for any $t \in[0, T]$ if $\nabla \cdot \boldsymbol{H}_{0}=0=\nabla \cdot \boldsymbol{F}(t)$ for any time $t \in[0, T]$, see Theorem 2. From now on, it is assumed that $\boldsymbol{H} \cdot \boldsymbol{\nu}=0$ on $\partial \Omega$. This is a natural 
condition due to (1.1) and the assumption that the magnetic field outside the domain equals zero [22, pp. 8]. Therefore, using the identity

$$
-\Delta \boldsymbol{H}=\nabla \times(\nabla \times \boldsymbol{H})-\nabla(\nabla \cdot \boldsymbol{H})
$$

and Lemma 3, the solution of problem (3.1) satisfies also

$$
\begin{cases}\partial_{t} \boldsymbol{H}-\Delta \boldsymbol{H}+\mathcal{K} \star \boldsymbol{H}=\boldsymbol{F} & \text { in } Q_{T} ; \\ \boldsymbol{H}=\mathbf{0} & \text { on } \partial \Omega \times(0, T) ; \\ \boldsymbol{H}(\boldsymbol{x}, 0)=\boldsymbol{H}_{0} & \text { in } \Omega ; \\ \nabla \cdot \boldsymbol{H}_{0}=\mathbf{0} & \text { in } \Omega .\end{cases}
$$

Therefore, our further analysis take place in the Hilbert spaces $\mathbf{H}^{1}(\Omega)$ and $\mathbf{H}^{2}(\Omega)$. First, a useful inequality is stated, which follows from Lemma 4, namely

$$
|\mathcal{K}(\boldsymbol{x}, \cdot) \star \boldsymbol{H}|=\left|\int_{\Omega} \mathcal{K}\left(\boldsymbol{x}, \boldsymbol{x}^{\prime}\right) \boldsymbol{H}\left(\boldsymbol{x}^{\prime}\right) \mathrm{d} \boldsymbol{x}^{\prime}\right| \leqslant C(q)\|\boldsymbol{H}\|_{q}, \quad \forall q>3, \quad \forall \boldsymbol{x} \in \Omega .
$$

Under the additional assumption that $\boldsymbol{H} \cdot \boldsymbol{\nu}=0$ on $\partial \Omega$, the solution to problem (3.1) obeys

$$
\left(\partial_{t} \boldsymbol{H}, \boldsymbol{\varphi}\right)+(\nabla \boldsymbol{H}, \nabla \boldsymbol{\varphi})+(\mathcal{K} \star \boldsymbol{H}, \boldsymbol{\varphi})=(\boldsymbol{F}, \boldsymbol{\varphi}), \quad \forall \boldsymbol{\varphi} \in \mathbf{H}_{0}^{1}(\Omega) .
$$

The following theorem is analogical to Theorem 2 .

Theorem 7 (Enhanced stability). Assume that $\nabla \cdot \boldsymbol{F}=0=\nabla \cdot \boldsymbol{H}_{0}, \boldsymbol{F} \in L^{2}\left((0, T), \mathbf{L}^{2}(\Omega)\right)$ and $\boldsymbol{H} \cdot \boldsymbol{\nu}=0$ on $\partial \Omega$. The solution to problem (3.1) obeys

(i) If $\boldsymbol{H}_{0} \in \mathbf{L}^{2}(\Omega)$ then

$$
\max _{t \in[0, T]}\|\boldsymbol{H}(t)\|^{2}+\int_{0}^{T}\|\nabla \boldsymbol{H}\|^{2} \leqslant C .
$$

(ii) Moreover, there exists a positive constant $C$ such that

$$
\int_{0}^{T}\left\|\partial_{t} \boldsymbol{H}\right\|_{\mathbf{H}^{-1}(\Omega)}^{2} \leqslant C
$$

(iii) If $\boldsymbol{H}_{0} \in \mathbf{H}_{0}^{1}(\Omega)$ then

$$
\max _{t \in[0, T]}\|\nabla \boldsymbol{H}(t)\|^{2}+\int_{0}^{T}\left\|\partial_{t} \boldsymbol{H}\right\|^{2} \leqslant C
$$

(iv) If $\boldsymbol{F}(0) \in \mathbf{L}^{2}(\Omega), \partial_{t} \boldsymbol{F} \in L^{2}\left((0, T), \mathbf{L}^{2}(\Omega)\right)$ and $\boldsymbol{H}_{0} \in \mathbf{H}_{0}^{1}(\Omega) \cap \mathbf{H}^{2}(\Omega)$ then

$$
\max _{t \in[0, T]}\left\|\partial_{t} \boldsymbol{H}(t)\right\|^{2}+\int_{0}^{T}\left\|\nabla \partial_{t} \boldsymbol{H}\right\|^{2} \leqslant C .
$$

Proof: The proof is straightforward if we follow the line from Theorem 2. Please note that we employ the positive definiteness of the convolution kernel $\mathcal{K}$ in order to avoid the use of Grönwall's lemma. We only point out how to handle the differences.

(i) Note that $\int_{0}^{t}(\mathcal{K} \star \boldsymbol{H}, \boldsymbol{H}) \geqslant 0$. Thanks to the embedding $\mathbf{H}_{0}^{1}(\Omega) \hookrightarrow \mathbf{L}^{2}(\Omega)$ (Rellich-Kondrachov Compactness Theorem, cf. [39, Theorem 1, p. 272]) and Friedrich's inequality (2.4), we get

$$
\int_{0}^{t}(\boldsymbol{F}, \boldsymbol{H}) \leqslant C_{\varepsilon} \int_{0}^{t}\|\boldsymbol{F}\|^{2}+\varepsilon \int_{0}^{t}\|\nabla \boldsymbol{H}\|^{2} .
$$


(ii) It holds

$$
\left(\partial_{t} \boldsymbol{H}, \boldsymbol{\varphi}\right)=(\boldsymbol{F}, \boldsymbol{\varphi})-(\nabla \boldsymbol{H}, \nabla \boldsymbol{\varphi})-(\mathcal{K} \star \boldsymbol{H}, \boldsymbol{\varphi}), \quad \boldsymbol{\varphi} \in \mathbf{H}_{0}^{1}(\Omega) .
$$

A simple calculation implies

$$
|(\boldsymbol{F}, \boldsymbol{\varphi})| \leqslant\|\boldsymbol{F}\|\|\boldsymbol{\varphi}\|, \quad|(\nabla \boldsymbol{H}, \nabla \boldsymbol{\varphi})| \leqslant\|\nabla \boldsymbol{H}\|\|\nabla \boldsymbol{\varphi}\|
$$

and using $\mathbf{H}_{0}^{1}(\Omega) \hookrightarrow \mathbf{L}^{6}(\Omega)$ (Sobolev embeddingstheorem in $\mathbb{R}^{3}$, cf. [22, Thm. 3.6]), we see that

$$
|(\mathcal{K} \star \boldsymbol{H}, \boldsymbol{\varphi})| \stackrel{(6.4)}{\lesssim}\|\nabla \boldsymbol{H}\|\|\boldsymbol{\varphi}\| .
$$

Thus using

$$
\left\|\partial_{t} \boldsymbol{H}\right\|_{\mathbf{H}^{-1}(\Omega)}=\sup _{\boldsymbol{\varphi} \in \mathbf{H}_{0}^{1}(\Omega)} \frac{\left(\partial_{t} \boldsymbol{H}, \boldsymbol{\varphi}\right)}{\|\boldsymbol{\varphi}\|_{\mathbf{H}_{0}^{1}(\Omega)}}
$$

we deduce that

$$
\int_{0}^{T}\left\|\partial_{t} \boldsymbol{H}\right\|_{\mathbf{H}^{-1}(\Omega)}^{2} \leqslant C
$$

(iii) According to $\mathbf{H}_{0}^{1}(\Omega) \hookrightarrow \mathbf{L}^{6}(\Omega)$, we successively deduce that

$$
\begin{aligned}
\int_{0}^{t}\left(\mathcal{K} \star \boldsymbol{H}, \partial_{t} \boldsymbol{H}\right) & \leqslant \varepsilon \int_{0}^{t}\left\|\partial_{t} \boldsymbol{H}\right\|^{2}+C_{\varepsilon} \int_{0}^{t}\|\mathcal{K} \star \boldsymbol{H}\|^{2} \\
& \stackrel{(6.4)}{\leqslant} \varepsilon \int_{0}^{t}\left\|\partial_{t} \boldsymbol{H}\right\|^{2}+C_{\varepsilon} \int_{0}^{t}\|\nabla \boldsymbol{H}\|^{2} \\
& \stackrel{(i)}{\leqslant} \varepsilon \int_{0}^{t}\left\|\partial_{t} \boldsymbol{H}\right\|^{2}+C_{\varepsilon} .
\end{aligned}
$$

(iv) Analogously as in Theorem 2(iv) we arrive at

$$
\max _{t \in[0, T]}\left\|\partial_{t} \boldsymbol{H}(t)\right\|^{2}+\int_{0}^{T}\left\|\nabla \partial_{t} \boldsymbol{H}\right\|^{2} \lesssim 1+\left\|\partial_{t} \boldsymbol{H}(0)\right\|^{2} .
$$

It holds

$$
\partial_{t} \boldsymbol{H}(0)=\boldsymbol{F}(0)-\Delta \boldsymbol{H}_{0}-\mathcal{K} \star \boldsymbol{H}_{0} .
$$

Taking into account $\mathbf{H}_{0}^{1}(\Omega) \hookrightarrow \mathbf{L}^{6}(\Omega)$ and (6.4) we get $\left\|\mathcal{K} \star \boldsymbol{H}_{0}\right\| \leqslant C$. Therefore

$$
\left\|\partial_{t} \boldsymbol{H}(0)\right\| \lesssim 1
$$

The implicit scheme (4.1) takes the form

$$
\left\{\begin{aligned}
\left(\delta \boldsymbol{h}_{i}, \boldsymbol{\varphi}\right)+\left(\nabla \boldsymbol{h}_{i}, \nabla \boldsymbol{\varphi}\right)+\left(\mathcal{K} \star \boldsymbol{h}_{i}, \boldsymbol{\varphi}\right) & =\left(\boldsymbol{f}_{i}, \boldsymbol{\varphi}\right), \quad \boldsymbol{\varphi} \in \mathbf{H}_{0}^{1}(\Omega) \\
\boldsymbol{h}_{0} & =\boldsymbol{H}_{0}
\end{aligned}\right.
$$

which is equivalent to

$$
\begin{aligned}
a\left(\boldsymbol{h}_{i}, \boldsymbol{\varphi}\right) & :=\left(\frac{\boldsymbol{h}_{i}}{\tau}, \boldsymbol{\varphi}\right)+\left(\nabla \boldsymbol{h}_{i}, \nabla \boldsymbol{\varphi}\right)+\left(\mathcal{K} \star \boldsymbol{h}_{i}, \boldsymbol{\varphi}\right) \\
& =\left(\boldsymbol{f}_{i}, \boldsymbol{\varphi}\right)+\left(\frac{\boldsymbol{h}_{i-1}}{\tau}, \boldsymbol{\varphi}\right)=: f_{i}(\boldsymbol{\varphi}) .
\end{aligned}
$$

The following lemma is analogous to Lemma 1. As in Theorem 7, the application of Grönwall's 
argument can be avoided by the positive definiteness of the kernel $\mathcal{K}$.

Lemma 6 (Enhanced a priori estimates). Assume that $\nabla \cdot \boldsymbol{F}=0=\nabla \cdot \boldsymbol{H}_{0}, \boldsymbol{F} \in L^{2}\left((0, T), \mathbf{L}^{2}(\Omega)\right)$ and $\boldsymbol{H} \cdot \boldsymbol{\nu}=0$ on $\partial \Omega$.

(i) Let $\boldsymbol{H}_{0} \in \mathbf{L}^{2}(\Omega)$. Then, there exists a positive constant $C$ such that

$$
\max _{1 \leqslant i \leqslant n}\left\|\boldsymbol{h}_{i}\right\|^{2}+\sum_{i=1}^{n}\left\|\boldsymbol{h}_{i}-\boldsymbol{h}_{i-1}\right\|^{2}+\sum_{i=1}^{n}\left\|\nabla \boldsymbol{h}_{i}\right\|^{2} \tau \leqslant C .
$$

(ii) There exists a positive constant $C$ such that

$$
\tau \sum_{i=1}^{n}\left\|\delta \boldsymbol{h}_{i}\right\|_{\mathbf{H}^{-1}(\Omega)}^{2} \leqslant C .
$$

(iii) If $\boldsymbol{H}_{0} \in \mathbf{H}_{0}^{1}(\Omega)$ then

$$
\max _{1 \leqslant i \leqslant n}\left\|\nabla \boldsymbol{h}_{i}\right\|^{2}+\sum_{i=1}^{n}\left\|\nabla \boldsymbol{h}_{i}-\nabla \boldsymbol{h}_{i-1}\right\|^{2}+\sum_{i=1}^{n}\left\|\delta \boldsymbol{h}_{i}\right\|^{2} \tau \leqslant C .
$$

(iv) If $\boldsymbol{F}(0) \in \mathbf{L}^{2}(\Omega), \partial_{t} \boldsymbol{F} \in L^{2}\left((0, T), \mathbf{L}^{2}(\Omega)\right)$ and $\boldsymbol{H}_{0} \in \mathbf{H}_{0}^{1}(\Omega) \cap \mathbf{H}^{2}(\Omega)$ then

$$
\max _{1 \leqslant i \leqslant n}\left\|\delta \boldsymbol{h}_{i}\right\|^{2}+\sum_{i=1}^{n}\left\|\delta \boldsymbol{h}_{i}-\delta \boldsymbol{h}_{i-1}\right\|^{2}+\sum_{i=1}^{n}\left\|\nabla \delta \boldsymbol{h}_{i}\right\|^{2} \tau \leqslant C .
$$

Therefore, the same stability results are obtained as in Lemma 1, where the curl-spaces are replaced by analogous $\mathbf{H}^{s}(\Omega)$-spaces. The variational formulation (6.6) can be rewritten in terms of the Rothe functions as

$$
\left(\partial_{t} \boldsymbol{H}_{n}(t), \boldsymbol{\varphi}\right)+\left(\nabla \overline{\boldsymbol{H}}_{n}(t), \nabla \boldsymbol{\varphi}\right)+\left(\mathcal{K} \star \overline{\boldsymbol{H}}_{n}(t), \boldsymbol{\varphi}\right)=\left(\overline{\boldsymbol{F}}_{n}(t), \boldsymbol{\varphi}\right), \quad \boldsymbol{\varphi} \in \mathbf{H}_{0}^{1}(\Omega) .
$$

Theorem 8 (Enhanced existence). Let $\boldsymbol{H}_{0} \in \mathbf{L}^{2}(\Omega)$ and $\boldsymbol{F} \in L^{2}\left((0, T), \mathbf{L}^{2}(\Omega)\right)$. Assume that $\nabla$. $\boldsymbol{H}_{0}=0=\nabla \cdot \boldsymbol{F}(t)$ for any time $t \in[0, T]$. If $\boldsymbol{H} \cdot \boldsymbol{\nu}=0$ on $\partial \Omega$, then the solution $\boldsymbol{H}$ to problem (3.1) belongs to $C\left([0, T], \mathbf{L}^{2}(\Omega)\right) \cap L^{2}\left((0, T), \mathbf{H}_{0}^{1}(\Omega)\right)$ with $\partial_{t} \boldsymbol{H} \in L^{2}\left((0, T), \mathbf{H}^{-1}(\Omega)\right)$.

Proof: The proof follows the same line as in Theorem 3. The main point of this theorem is the embedding

$$
\mathbf{H}_{0}^{1}(\Omega) \hookrightarrow \hookrightarrow \mathbf{L}^{2}(\Omega) \cong \mathbf{L}^{2}(\Omega)^{*} \hookrightarrow \mathbf{H}^{-1}(\Omega) .
$$

Lemma $6(i)$ and (ii) give $\boldsymbol{H}_{n} \in L^{2}\left((0, T), \mathbf{H}_{0}^{1}(\Omega)\right)$. Using $\partial_{t} \boldsymbol{H}_{n} \in L^{2}\left((0, T), \mathbf{H}^{-1}(\Omega)\right)$ and the generalized Aubin-Lions lemma [32, Lemma 7.7], we get that $\left\{\boldsymbol{H}_{n}\right\}$ is compact in the space $L_{2}\left((0, T), \mathbf{L}^{2}(\Omega)\right)$. Therefore, there exists a subsequence of $\boldsymbol{H}_{n}$ (denoted by the same symbol again) for which we have (cf. [33, p. 88])

$$
\boldsymbol{H}_{n}(\boldsymbol{x}, t) \rightarrow \boldsymbol{H}(\boldsymbol{x}, t) \quad \text { for a.e. }(\boldsymbol{x}, t) \in Q_{T} .
$$

Moreover, $\boldsymbol{H}_{n} \rightarrow \boldsymbol{H}$ in $L^{2}\left((0, T), \mathbf{H}_{0}^{1}(\Omega)\right)$. Applying [32, Lemma 7.3], we get $\boldsymbol{H} \in C\left([0, T], \mathbf{L}^{2}(\Omega)\right)$, which concludes the proof.

The following error estimates have smaller constant $C$ in comparison with the constants appearing in Theorem 4 because Grönwall's argument is avoided.

Theorem 9 (Error). Assume that $\boldsymbol{F} \in \operatorname{Lip}\left([0, T], \mathbf{L}^{2}(\Omega)\right)$. 
(i) If $\boldsymbol{H}_{0} \in \mathbf{H}_{0}^{1}(\Omega)$ then

$$
\max _{t \in[0, T]}\left\|\boldsymbol{H}_{n}(t)-\boldsymbol{H}(t)\right\|^{2}+\int_{0}^{T}\left\|\nabla\left[\boldsymbol{H}_{n}-\boldsymbol{H}\right]\right\|^{2} \leqslant C \tau .
$$

(ii) If $\boldsymbol{H}_{0} \in \mathbf{H}_{0}^{1}(\Omega) \cap \mathbf{H}^{2}(\Omega)$ then

$$
\max _{t \in[0, T]}\left\|\boldsymbol{H}_{n}(t)-\boldsymbol{H}(t)\right\|^{2}+\int_{0}^{T}\left\|\nabla\left[\boldsymbol{H}_{n}-\boldsymbol{H}\right]\right\|^{2} \leqslant C \tau^{2} .
$$

Proof: We subtract (6.5) from (6.7), set $\boldsymbol{\varphi}=\boldsymbol{H}_{n}-\boldsymbol{H}$ and integrate in time to get

$$
\begin{gathered}
\frac{1}{2}\left\|\boldsymbol{H}_{n}(t)-\boldsymbol{H}(t)\right\|^{2}+\int_{0}^{t}\left\|\nabla\left[\boldsymbol{H}_{n}-\boldsymbol{H}\right]\right\|^{2}+\int_{0}^{t}\left(\mathcal{K} \star\left[\boldsymbol{H}_{n}-\boldsymbol{H}\right], \boldsymbol{H}_{n}-\boldsymbol{H}\right) \\
=\int_{0}^{t}\left(\overline{\boldsymbol{F}}_{n}-\boldsymbol{F}, \boldsymbol{H}_{n}-\boldsymbol{H}\right)+\int_{0}^{t}\left(\nabla\left[\boldsymbol{H}_{n}-\overline{\boldsymbol{H}}_{n}\right], \nabla\left[\boldsymbol{H}_{n}-\boldsymbol{H}\right]\right) \\
+\int_{0}^{t}\left(\mathcal{K} \star\left[\boldsymbol{H}_{n}-\overline{\boldsymbol{H}}_{n}\right], \boldsymbol{H}_{n}-\boldsymbol{H}\right) .
\end{gathered}
$$

The last term on the LHS is non-negative. Further

$\left|\int_{0}^{t}\left(\overline{\boldsymbol{F}}_{n}-\boldsymbol{F}, \boldsymbol{H}_{n}-\boldsymbol{H}\right)\right| \leqslant C_{\varepsilon} \int_{0}^{t}\left\|\overline{\boldsymbol{F}}_{n}-\boldsymbol{F}\right\|^{2}+\varepsilon \int_{0}^{t}\left\|\boldsymbol{H}_{n}-\boldsymbol{H}\right\|^{2} \leqslant C_{\varepsilon} \tau^{2}+\varepsilon \int_{0}^{t}\left\|\nabla\left[\boldsymbol{H}_{n}-\boldsymbol{H}\right]\right\|^{2}$.

It holds

$$
\left\|\nabla\left[\boldsymbol{H}_{n}(t)-\overline{\boldsymbol{H}}_{n}(t)\right]\right\| \leqslant \tau\left\|\partial_{t} \nabla \boldsymbol{H}_{n}(t)\right\| \quad \text { for } t \in[0, T] .
$$

For the last term of (6.8) we may write using (6.4) and $\mathbf{H}_{0}^{1}(\Omega) \hookrightarrow \mathbf{L}^{6}(\Omega)$ that

$$
\begin{aligned}
\left|\int_{0}^{t}\left(\mathcal{K} \star\left[\boldsymbol{H}_{n}-\overline{\boldsymbol{H}}_{n}\right], \boldsymbol{H}_{n}-\boldsymbol{H}\right)\right| & \lesssim \int_{0}^{t}\left\|\nabla\left[\boldsymbol{H}_{n}-\overline{\boldsymbol{H}}_{n}\right]\right\|\left\|\boldsymbol{H}_{n}-\boldsymbol{H}\right\| \\
& \leqslant \varepsilon \int_{0}^{t}\left\|\boldsymbol{H}_{n}-\boldsymbol{H}\right\|^{2}+C_{\varepsilon} \int_{0}^{t}\left\|\nabla\left[\boldsymbol{H}_{n}-\overline{\boldsymbol{H}}_{n}\right]\right\|^{2} \\
& \leqslant \varepsilon \int_{0}^{t}\left\|\nabla\left[\boldsymbol{H}_{n}-\boldsymbol{H}\right]\right\|^{2}+C_{\varepsilon} \int_{0}^{t}\left\|\nabla\left[\boldsymbol{H}_{n}-\overline{\boldsymbol{H}}_{n}\right]\right\|^{2} .
\end{aligned}
$$

Thus, we see employing Lemma $6(i i i)$ and $(i v)$ that
(i) $\left|\int_{0}^{t}\left(\mathcal{K} \star\left[\boldsymbol{H}_{n}-\overline{\boldsymbol{H}}_{n}\right], \boldsymbol{H}_{n}-\boldsymbol{H}\right)\right| \leqslant \varepsilon \int_{0}^{t}\left\|\nabla\left[\boldsymbol{H}_{n}-\boldsymbol{H}\right]\right\|^{2}+C_{\varepsilon} \tau$
(ii) $\left|\int_{0}^{t}\left(\mathcal{K} \star\left[\boldsymbol{H}_{n}-\overline{\boldsymbol{H}}_{n}\right], \boldsymbol{H}_{n}-\boldsymbol{H}\right)\right| \leqslant \varepsilon \int_{0}^{t}\left\|\nabla\left[\boldsymbol{H}_{n}-\boldsymbol{H}\right]\right\|^{2}+C_{\varepsilon} \tau^{2}$.

It remains to estimate the second term on the RHS in (6.8). Depending on the stability results we have

$$
\begin{aligned}
\left|\int_{0}^{t}\left(\nabla\left[\boldsymbol{H}_{n}-\overline{\boldsymbol{H}}_{n}\right], \nabla\left[\boldsymbol{H}_{n}-\boldsymbol{H}\right]\right)\right| & \leqslant \varepsilon \int_{0}^{t}\left\|\nabla\left[\boldsymbol{H}_{n}-\boldsymbol{H}\right]\right\|^{2}+C_{\varepsilon} \int_{0}^{t}\left\|\nabla\left[\boldsymbol{H}_{n}-\overline{\boldsymbol{H}}_{n}\right]\right\|^{2} \\
& \leqslant \varepsilon \int_{0}^{t}\left\|\nabla\left[\boldsymbol{H}_{n}-\boldsymbol{H}\right]\right\|^{2}+C_{\varepsilon} \tau
\end{aligned}
$$

$$
\begin{aligned}
\left|\int_{0}^{t}\left(\nabla\left[\boldsymbol{H}_{n}-\overline{\boldsymbol{H}}_{n}\right], \nabla\left[\boldsymbol{H}_{n}-\boldsymbol{H}\right]\right)\right| & \leqslant \varepsilon \int_{0}^{t}\left\|\nabla\left[\boldsymbol{H}_{n}-\boldsymbol{H}\right]\right\|^{2}+C_{\varepsilon} \int_{0}^{t}\left\|\nabla\left[\boldsymbol{H}_{n}-\overline{\boldsymbol{H}}_{n}\right]\right\|^{2} \\
& \leqslant \varepsilon \int_{0}^{t}\left\|\nabla\left[\boldsymbol{H}_{n}-\boldsymbol{H}\right]\right\|^{2}+C_{\varepsilon} \tau^{2}
\end{aligned}
$$

Putting things together and choosing a sufficiently small positive $\varepsilon$, we conclude the proof. 


\subsection{Modified scheme in $\mathbf{H}^{1}(\Omega)$}

Finally, the following semi-implicit time-discrete scheme is considered, which represents a slight modification of (6.6). Here, the convolution term is taken explicitly (from the last time step)

$$
\left\{\begin{aligned}
\left(\delta \boldsymbol{h}_{i}, \boldsymbol{\varphi}\right)+\left(\nabla \boldsymbol{h}_{i}, \nabla \boldsymbol{\varphi}\right) & =\left(\boldsymbol{f}_{i}, \boldsymbol{\varphi}\right)-\left(\mathcal{K} \star \boldsymbol{h}_{i-1}, \boldsymbol{\varphi}\right), \quad \boldsymbol{\varphi} \in \mathbf{H}_{0}^{1}(\Omega) \\
\boldsymbol{h}_{0} & =\boldsymbol{H}_{0}
\end{aligned}\right.
$$

which is equivalent to

$$
\begin{aligned}
a\left(\boldsymbol{h}_{i}, \boldsymbol{\varphi}\right) & :=\left(\frac{\boldsymbol{h}_{i}}{\tau}, \boldsymbol{\varphi}\right)+\left(\nabla \boldsymbol{h}_{i}, \nabla \boldsymbol{\varphi}\right) \\
& =\left(\boldsymbol{f}_{i}, \boldsymbol{\varphi}\right)-\left(\mathcal{K} \star \boldsymbol{h}_{i-1}, \boldsymbol{\varphi}\right)+\left(\frac{\boldsymbol{h}_{i-1}}{\tau}, \boldsymbol{\varphi}\right)=: f_{i}(\boldsymbol{\varphi}) .
\end{aligned}
$$

The existence of a unique solution is obtained to (6.9) for any $i=1, \ldots, n$ and any $\tau>0$ if $\boldsymbol{H}_{0} \in \mathbf{L}^{q}(\Omega), q>3$. The scheme (6.9) can be analysed in the same way as (6.6), therefore, further details are omitted. However, the use of Grönwall's lemma with exponential in time character of the constant cannot be avoided despite of the positive definiteness of $\mathcal{K}$. Nevertheless, the error estimates from Theorem 9 are also valid for (6.9). The main difference is that the constants $C$ will be larger. In the following section, the convergence of this scheme is illustrated in some numerical experiments. Future research can concern the implementation of the other schemes. This section is concluded with a comparison of the obtained results with the London equations.

\subsection{Comparison with the London equations}

Consider a thin superconducting slab, of thickness $2 L$, as shown in Fig. 1. The second London

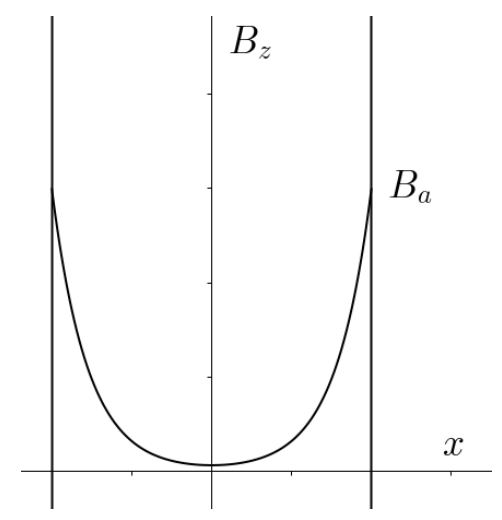

Figure 1: The magnetic field inside a superconducting slab of thickness $2 L$.

equation is given by $\nabla \times \boldsymbol{J}_{s}=-\Lambda^{-1} \boldsymbol{B}$. This equation, combined with the quasi-static Maxwell equation $\nabla \times \boldsymbol{H}=\boldsymbol{J}_{s}$ and equation (1.1) gives

$$
\nabla \times \nabla \times \boldsymbol{B}=-\frac{1}{\beta} \boldsymbol{B}
$$

where $\beta:=\frac{\Lambda}{\mu}=\frac{m_{e}}{\mu n_{s} e^{2}}$. But $\nabla \times(\nabla \times \boldsymbol{B})=\nabla(\nabla \cdot \boldsymbol{B})-\Delta \boldsymbol{B}=-\Delta \boldsymbol{B}$ since $\nabla \cdot \boldsymbol{B}=0$. Hence,

$$
\Delta \boldsymbol{B}=\frac{1}{\beta} \boldsymbol{B}
$$

Suppose that an external parallel magnetic field is applied in the $z$ direction parallel to the slab surfaces, $\boldsymbol{B}=\left(0,0, B_{a}\right)$. Given that inside the superconductor the magnetic field is a function of $x$ only, $\boldsymbol{B}=$ 
$\left(0,0, B_{z}(x)\right)$, equation (6.10) is equivalent with

$$
\frac{d^{2} B_{z}(x)}{d x^{2}}=\frac{1}{\beta} B_{z}(x)
$$

Solving equation (6.11) with the boundary conditions that $B_{z}=B_{a}$ at the two surfaces at $x= \pm L$, the solution inside the slab becomes

$$
B_{z}(x)=B_{a} \frac{\cosh \left(\frac{x}{\sqrt{\beta}}\right)}{\cosh \left(\frac{L}{\sqrt{\beta}}\right)} .
$$

This result shows that a magnetic field is exponentially decayed at the surface of a superconductor (Meissner effect). The magnetic field $\boldsymbol{B}$ penetrates at the surfaces corresponding with $x= \pm L$ approximately over a distance $\sqrt{\beta}$ (London penetration depth). Analogue, choosing $\boldsymbol{J}_{n}=\mathbf{0}$ in the calculation of model (6.3), the magnetic field $\boldsymbol{H}$ statifies the following elliptic integro-differential equation for type-I superconductivity

$$
\Delta \boldsymbol{H}=\mathcal{K} \star \boldsymbol{H} \quad \text { or } \quad \Delta \boldsymbol{B}=\mathcal{K} \star \boldsymbol{B} .
$$

If $r_{0} \rightarrow 0$, thus the support of $\mathcal{K}$ becomes smaller and smaller, $\mathcal{K}$ becomes a Dirac delta function. Consequently, $\mathcal{K} \rightarrow \frac{\widetilde{C}}{2} \delta\left(\boldsymbol{x}-\boldsymbol{x}^{\prime}\right)$ and (6.13) converts to

$$
\Delta \boldsymbol{B}=\frac{\widetilde{C}}{2} \boldsymbol{B}
$$

The identification $\frac{\widetilde{C}}{2}=\frac{\mu}{\Lambda}=\frac{1}{\beta}$ yields the London equation (6.10). An interesting area for future research is to compare the numerical solution of problem (6.13) for small $r_{0}$ with the exact solution (6.12) in the case of an infinite slab.

\section{Numerical experiments}

For the numerical experiments is assumed that the domain $\Omega \subset \mathbb{R}^{3}$ is a Lipschitz polyhedron. Consider a triangulation of the domain $\Omega$. Hence, there exists a finite set of tetrahedra $\mathcal{T}_{h}$ such that $\bar{\Omega}=\bigcup_{\mathcal{T} \in \mathcal{T}_{h}} \overline{\mathcal{T}}$. Remark that $h=\max _{\mathcal{T} \in \mathcal{T}_{h}} h_{\mathcal{T}}$, where $h_{\mathcal{T}}$ is the diameter of the smallest sphere containing $\mathcal{T}$. The total number of vertices is setted equal to $M$ and the $i$ th vertex of $\mathcal{T}_{h}$ is put equal to $\boldsymbol{x}_{i}$. Denote by $\boldsymbol{x}_{m, \mathcal{T}}$ and $\operatorname{Vol}(\mathcal{T})$ the midpoint and the volume respectively of a tetrahedron $\mathcal{T} \in \mathcal{T}_{h}$. Define the set

$$
\mathcal{T}_{\boldsymbol{x}}:=\left\{\mathcal{T} \in \mathcal{T}_{h}:\left|\boldsymbol{x}_{m, \mathcal{T}}-\boldsymbol{x}\right|<r_{0}\right\} \subset \mathcal{T}_{h}
$$

The convolution integral arising in the numerical experiments is solved numerically. Namely,

$$
\mathcal{C}(\boldsymbol{x}, \varphi):=\mathcal{K}(\boldsymbol{x}, \cdot) \star \varphi \approx \sum_{\mathcal{T} \in \mathcal{T}_{\boldsymbol{x}}} \operatorname{Vol}(\mathcal{T}) \mathcal{K}\left(\boldsymbol{x}-\boldsymbol{x}_{m, \mathcal{T}}\right) \boldsymbol{\varphi}\left(\boldsymbol{x}_{m, \mathcal{T}}\right)
$$

This is a way to avoid the singularity in the kernel. In this section, the numerical solution of problem (6.3) is derived. The time domain is discretized using an equidistant time partitioning with time step $\tau=2^{-j}, 2 \leqslant j \leqslant 7$. The backward Euler difference method is applied. The following scheme is followed, see (6.9):

$$
\left\{\begin{aligned}
\left(\boldsymbol{h}_{i}, \boldsymbol{\varphi}\right)+\tau\left(\nabla \boldsymbol{h}_{i}, \nabla \boldsymbol{\varphi}\right) & =\tau\left(\boldsymbol{f}_{i}, \boldsymbol{\varphi}\right)-\tau\left(\mathcal{K} \star \boldsymbol{h}_{i-1}, \boldsymbol{\varphi}\right)+\left(\boldsymbol{h}_{i-1}, \boldsymbol{\varphi}\right), \quad \boldsymbol{\varphi} \in \mathbf{H}_{0}^{1}(\Omega), \\
\boldsymbol{h}_{0} & =\boldsymbol{H}_{0},
\end{aligned}\right.
$$


$i=1, \ldots, 2^{j} T$. The term $\left(\mathcal{K} \star \boldsymbol{h}_{i-1}, \boldsymbol{\varphi}\right)$ in the variational formulation can be considered as

$$
\int_{\Omega} \mathcal{C}\left(\boldsymbol{x}, \boldsymbol{h}_{i-1}\right) \cdot \boldsymbol{\varphi}(\boldsymbol{x}) \mathrm{d} \boldsymbol{x}
$$

At each time step, the resulting elliptic BVP (7.2) is solved numerically by the finite element method (FEM) using first first-order Lagrange elements on tetrahedra. The basis functions $\left\{\varphi_{j}\right\}_{j=1}^{M}$, such that

$$
\varphi_{j}\left(\boldsymbol{x}_{i}\right)=\left\{\begin{array}{ll}
1 & i=j ; \\
0 & i \neq j ;
\end{array} \quad i, j=1, \ldots M\right.
$$

span the finite element space $\mathcal{V}_{h} \subset H^{1}(\Omega)$. Therefore, for $\Psi \in \mathcal{V}_{h}$ holds

$$
\Psi(\boldsymbol{x})=\sum_{j=1}^{M} \Psi_{j} \varphi_{j}(\boldsymbol{x}), \quad \boldsymbol{x} \in \Omega,
$$

where $\Psi_{j} \approx \Psi\left(\boldsymbol{x}_{j}\right)$. For a vector field, each component is interpolated separately using the given basis functions. Define

$$
\boldsymbol{h}_{i}=\left(\begin{array}{l}
\sum_{j=1}^{M} c_{j}^{i} \varphi_{j} \\
\sum_{j=1}^{M} d_{j}^{i} \varphi_{j} \\
\sum_{j=1}^{M} e_{j}^{i} \varphi_{j}
\end{array}\right), \quad i=1, \ldots, 2^{j} T .
$$

The values $c_{j}^{i}, d_{j}^{i}, e_{j}^{i}$ for $j=1, \ldots, M$ are known as the degrees of freedom of $\boldsymbol{h}_{i}$. On each time step, an algebraic system is solved of the form

$$
A H^{i}=b^{i},
$$

where $H^{i}=\left(\boldsymbol{c}^{i} \boldsymbol{d}^{i} \boldsymbol{e}^{i}\right)^{T}$. The algorithm for the determination of the form of the matrix $A$ and the vector $b^{i}$ is written down. The finite element library DOLFIN from the FEniCS project is used for the implementation [40]. Remark that the matrix $A$ is time independent and can be computed before the time stepping.

\section{Algorithm.}

- Choose $\widetilde{C}$ and $r_{0}$. Prescribe an exact solution $\boldsymbol{H}^{\mathrm{ex}}$;

- $t=\tau$;

- Define separate forms $a_{M}(\boldsymbol{u}, \boldsymbol{v})=(\boldsymbol{u}, \boldsymbol{v})$ and $a_{S}(\boldsymbol{u}, \boldsymbol{v})=(\nabla \boldsymbol{u}, \nabla \boldsymbol{v})$;

- Assemble $a_{M}$ to the mass matrix $M$ and $a_{S}$ to the stiffness matrix $S$;

- Compute $A=M+\tau S$;

- While $t \leqslant T$ :

- Interpolate the solution on the previous time step, $\boldsymbol{h}_{i-1}$, componentwise to a finite element function $H^{i-1}$ in $\mathcal{V}_{h}:=\left(\mathcal{V}_{h}\right)^{3}$;

- Interpolate $\mathcal{C}\left(\boldsymbol{x}, \boldsymbol{h}_{i-1}\right)$ componentwise to a finite element function $\mathcal{C}_{p}$ in $\mathcal{V}_{h}$;

- Interpolate the formula for $\boldsymbol{F}=\partial_{t} \boldsymbol{H}^{\mathrm{ex}}-\Delta \boldsymbol{H}^{\mathrm{ex}}+K \star \boldsymbol{H}^{\mathrm{ex}}$ on time step $t_{i}$, i.e. $\boldsymbol{F}_{i}$, componentwise to a finite element function $F^{i}$ in $\mathcal{V}_{h}$. The convolution $K(\boldsymbol{x}, \cdot) \star \boldsymbol{h}_{i}^{\text {ex }}$ can be approximated by $\mathcal{C}\left(\boldsymbol{x}, \boldsymbol{h}_{i}^{\mathrm{ex}}\right)$.

- $b^{i}=\tau M F^{i}-\tau M \mathcal{C}_{p}+M H^{i-1}$;

- Solve $A H^{i}=b^{i}$ for $H^{i}$ and store in $\boldsymbol{h}_{i}$;

$-t \leftarrow t+\tau$;

$-\boldsymbol{h}_{i-1} \leftarrow \boldsymbol{h}_{i}$. 
The following assumptions are made in the experiments: $T=1, \Omega=[0,1] \times[0,1] \times[0,1]$ and $r_{0}=0.1$. Moreover, two values for the parameter $\widetilde{C}$ are used, namely $\widetilde{C}=2$ and $\widetilde{C}=150$. For the space discretization, a fixed uniform mesh is needed that gives a good approximation for the convolution integral. The number of cells in each direction is choosen to be equal, namely $\left(n_{x}, n_{x}, n_{x}\right)$. The volume of the sphere $S$ with center $\boldsymbol{x}$ and radius $r_{0}$, denoted by $\operatorname{Vol}(S)$, is compared with the volume of the set $\mathcal{T}_{\boldsymbol{x}}$ that is defined by

$$
\operatorname{Vol}\left(\mathcal{T}_{\boldsymbol{x}}\right)=\sum_{\mathcal{T} \in \mathcal{T}_{\boldsymbol{x}}} \operatorname{Vol}(\mathcal{T})
$$

where $\boldsymbol{x}$ a node is in $\left[r_{0}, 1-r_{0}\right] \times\left[r_{0}, 1-r_{0}\right] \times\left[r_{0}, 1-r_{0}\right]$. The results are given in Table 1 . The best approximation for $n_{x} \leqslant 30$ is 25 . For this reason, the total number of tetrahedra in the experiments will be 93750 . For every time step $\tau$, the error

$$
E=\max _{t \in[0, T]}\left\|\boldsymbol{h}_{n}(t)-\boldsymbol{H}(t)\right\|^{2}
$$

is computed. For all experiments, the exact solution belongs to $\mathcal{V}_{h}$. Therefore, the error is only due to the time discretization and the approximation of the convolution. Note that in the numerical experiments, the assumptions $\boldsymbol{H} \times \boldsymbol{\nu}=\mathbf{0}$ and $\boldsymbol{H} \cdot \boldsymbol{\nu}=\mathbf{0}$ do not need to be satisfied. The proposed schemes are also valid for a more general boundary condition.

\subsection{Experiment 1}

In the first experiment,

$$
\boldsymbol{H}^{\mathrm{ex}}=\left(1+t^{2}\right)\left(\begin{array}{l}
1 \\
1 \\
1
\end{array}\right)
$$

is used as exact solution. The RHS $\boldsymbol{F}$ can be calculated exactly in $\left[r_{0}, 1-r_{0}\right] \times\left[r_{0}, 1-r_{0}\right] \times\left[r_{0}, 1-r_{0}\right]$, namely

$$
\boldsymbol{F}^{\mathrm{ex}}=2 t+\left(2+2 t^{2}\right) \pi \widetilde{C} r_{0} \exp (-1.0)\left(\begin{array}{l}
1 \\
1 \\
1
\end{array}\right) \text {. }
$$

The error (7.3) is computed for $\Omega=\left[r_{0}, 1-r_{0}\right] \times\left[r_{0}, 1-r_{0}\right] \times\left[r_{0}, 1-r_{0}\right]$ and $\tau=2^{-j}, 2 \leqslant j \leqslant 7$, and is depicted in Figure 2 for $\widetilde{C}=2$ and $\widetilde{C}=150$. The error $\log _{2} E$ is plotted as a function of $\log _{2} \tau$. The expected convergence rate for smooth functions is predicted in Theorem 9: $E \sim \mathcal{O}\left(\tau^{2}\right)$. The linear regression line for the first two data points are given by $\log _{2} E=0.8881 \log _{2} \tau-11.4836$ and $\log _{2} E=$ $0.4752 \log _{2} \tau-2.1577$ for $\widetilde{C}=2$, respectively $\widetilde{C}=150$. Therefore, the expected linear behaviour is not obtained. With decreasing time step, the error in the approximation of the convolution starts dominating over the time discretization error. But, the error in the approximation of the convolution is sufficiently small. In the following two experiments, the error due to the numerical convolution is cancelled out because the numerical convolution is also used in the determination of $\boldsymbol{F}$.

\subsection{Experiment 2}

In this experiment, the following exact solution is defined

$$
\boldsymbol{H}^{\mathrm{ex}}=(1+t)\left(\begin{array}{l}
y-z \\
z-x \\
x-y
\end{array}\right) .
$$

The error (7.3) is again computed for $\tau=2^{-j}, 2 \leqslant j \leqslant 7$, and is shown in Figure 3 for $\widetilde{C}=2$ and $\widetilde{C}=150$. Now, a linear regression line is calculated through all the obtained data points: $\log _{2} E=$ $2 \log _{2} \tau-17.521$ and $\log _{2} E=2.148 \log _{2} \tau-5.0102$ for $\widetilde{C}=2$, respectively $\widetilde{C}=150$. This is in accordance with the predicted convergence rate $\mathcal{O}\left(\tau^{2}\right)$ in Theorem 9 . 


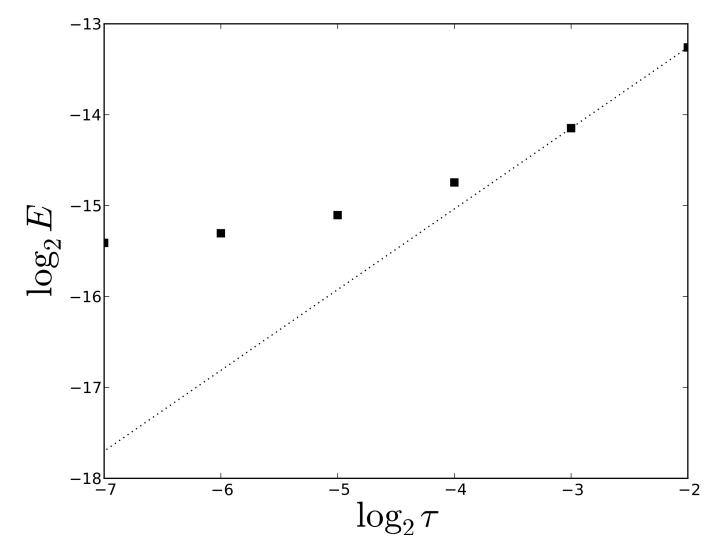

(a) $\widetilde{C}=2$

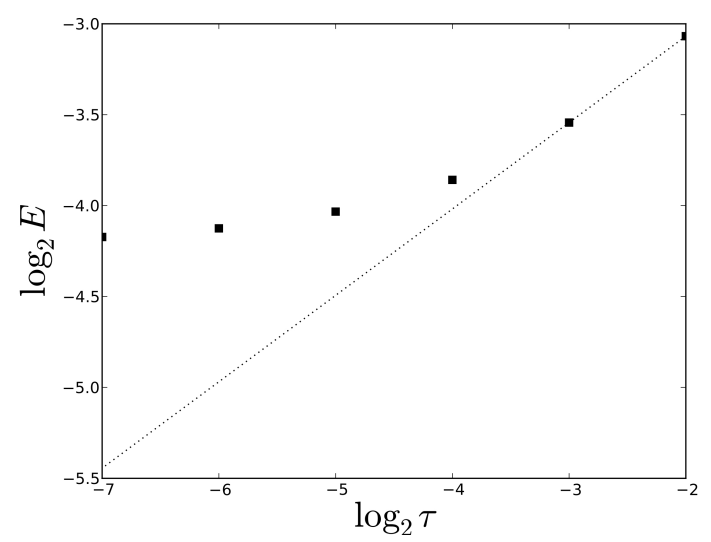

(b) $\widetilde{C}=150$

Figure 2: Results of numerical experiment 1. (a) convergence rate for experiment 1 with $\widetilde{C}=2$. (b) convergence rate for experiment 1 with $\widetilde{C}=150$.

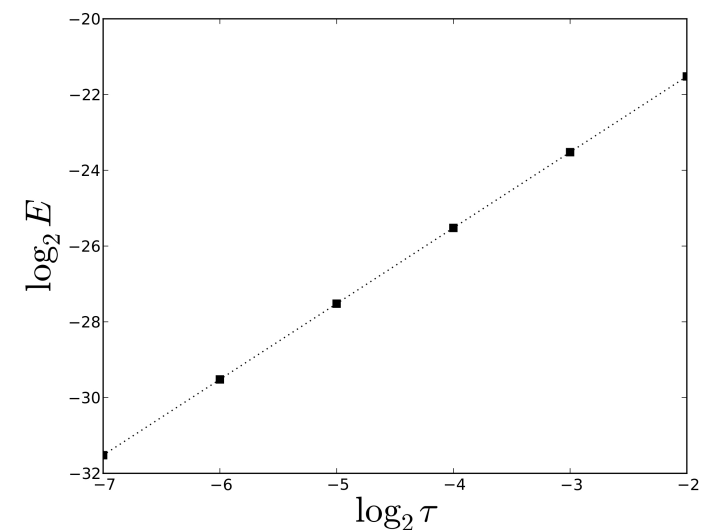

(a) $\widetilde{C}=2$

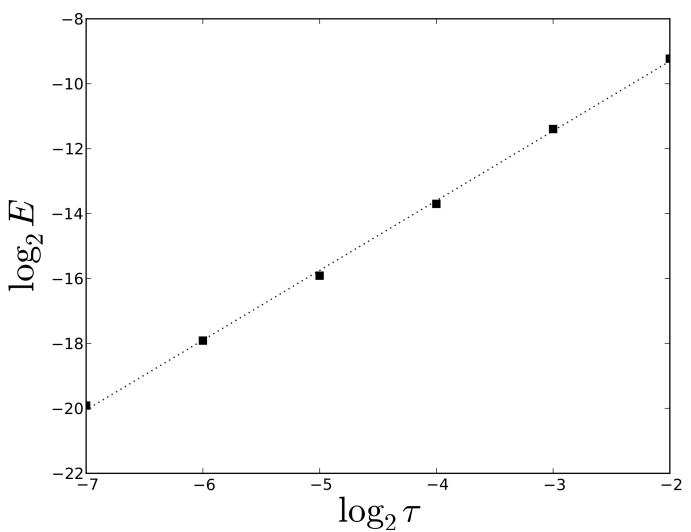

(b) $\widetilde{C}=150$

Figure 3: Results of numerical experiment 2. (a) convergence rate for experiment 2 with $\widetilde{C}=2$. (b) convergence rate for experiment 2 with $\widetilde{C}=150$. 


\subsection{Experiment 3}

In the last experiment, the following exact solution is taken

$$
\boldsymbol{H}^{\mathrm{ex}}=\left(1+t^{2}\right)\left(\begin{array}{l}
y-z \\
z-x \\
x-y
\end{array}\right) .
$$

The linear regression lines are $\log _{2} E=1.9753 \log _{2} \tau-12.858$ and $\log _{2} E=1.9678 \log _{2} \tau-4.0842$ for $\widetilde{C}=2$, respectively $\widetilde{C}=150$, see Figure 4 . The predicted convergence rate $\mathcal{O}\left(\tau^{2}\right)$ is not exactly obtained since the exact solution is quadratic in time.

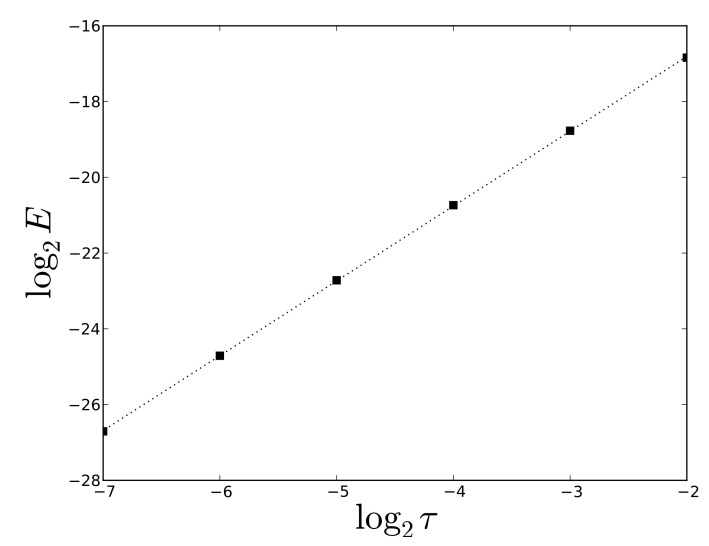

(a) $\widetilde{C}=2$

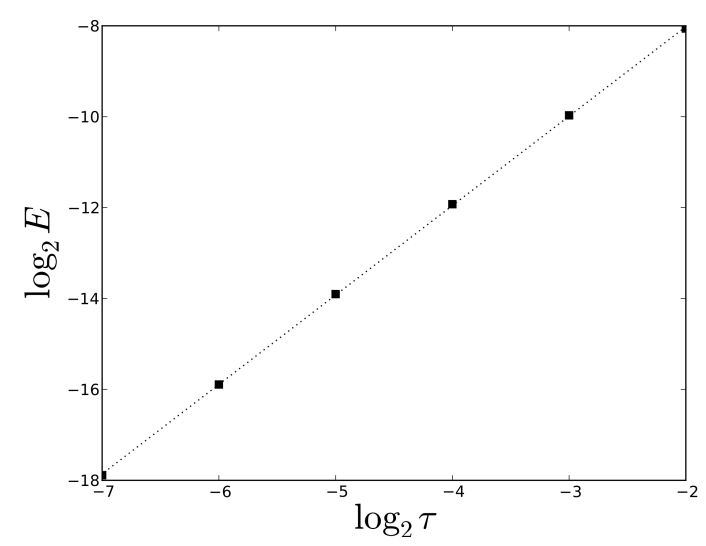

(b) $\widetilde{C}=150$

Figure 4: Results of numerical experiment 3. (a) convergence rate for experiment 3 with $\widetilde{C}=2$. (b) convergence rate for experiment 3 with $\widetilde{C}=150$.

\section{Conclusion}

A vectorial nonlocal linear parabolic problem (3.1) in terms of the magnetic field with applications in superconductors of type-I is studied. This model is obtained from the eddy current version of the Maxwell equations, the two-fluid model of London and London, and the nonlocal representation of the superconductive current by Eringen. The nonlocal term is given by a space convolution with a singular kernel. Two time-discrete schemes, a semi-implicit and an implicit (the convolution is taken explicit and implicit), are established. These schemes are also based on the backward Euler method. The well-posedness of the problem is shown under low regularity assumptions and the error estimates are derived for the two time-discrete schemes. Afterwards, a new convolution kernel is derived under the assumption that the normal component of the unknown vector field equals zero on the boundary of the superconductor. The positive definiteness of this kernel is shown. Using the obtained expression, it is demonstrated that the solution of the original model satisfies a simpler problem which is easier to implement. Both time-discrete schemes stay valid. Better error estimates are obtained for the implicit scheme. Finally, a numerical experiment for the semi-implicit scheme supports the obtained theoretical results.

Acknowledgment. The authors are supported by the IAP P7/02-project of the Belgian Science Policy. The computational resources (Stevin Supercomputer Infrastructure) and services used in this work were provided by the VSC (Flemish Supercomputer Center), funded by Ghent University, the Hercules Foundation and the Flemish Government - department EWI. The authors thank the reviewers for closely reading the manuscript and their useful suggestions. 


\section{References}

[1] J. Bardeen, L. N. Cooper, and J. R. Schrieffer. Microscopic theory of superconductivity. Phys. Rev., 106:162-164, Apr 1957.

[2] J. G. Bednorz and K. A. Müller. Possible high $\mathrm{T}_{c}$ superconductivity in the Ba-La-Cu-O system. Zeitschrift fur Physik B Condensed Matter, 64:189-193, June 1986.

[3] C. P. Bean. Magnetization of high-field superconductors. Rev. Mod. Phys., 36:31-39, Jan 1964.

[4] J.W. Barrett and L. Prigozhin. Beans critical-state model as the $p \rightarrow \infty$ limit of an evolutionary $p$-laplacian equation. Nonlinear Anal. Theory Methods Appl, 6, 2000.

[5] H.-M. Yin, B. Li, and J. Zou. A degenerate evolution system modeling bean's critical-state type-II superconductors. Discrete and Continuous Dynamical Systems, 8, 2002.

[6] L. Prigozhin and V. Sokolovsky. Ac losses in type-II superconductors induced by nonuniform fluctuations of external magnetic field. IEEE Transactions on Applied Superconductivity, 14, 2004.

[7] W. Wei and H.-M. Yin. Numerical solutions to bean's critical-state model for type-II superconductors. International Journal of Numerical Analysis and Modeling, 2, 2005.

[8] J. Rhyner. Magnetic properties and ac-losses of superconductors with power law currentvoltage characteristics. Physica C: Superconductivity, 212(34):292 - 300, 1993.

[9] E. H. Brandt. Superconductors of finite thickness in a perpendicular magnetic field: Strips and slabs. Phys. Rev. B, 54:4246-4264, Aug 1996.

[10] A. Gurevich and M. Friesen. Nonlinear transport current flow in superconductors with planar obstacles. Phys. Rev. B, 62:4004-4025, Aug 2000.

[11] M. Slodička. Nonlinear diffusion in type-II superconductors. J. Comput. Appl. Math., 215(2):568576, May 2008.

[12] A. B. Pippard. An experimental and theoretical study of the relation between magnetic field and current in a superconductor. Proceedings of the Royal Society of London. Series A, Mathematical and Physical Sciences, 216(1127):547-568, 1953.

[13] A.C. Eringen. Electrodynamics of memory-dependent nonlocal elastic continua. J. Math. Phys., 25:3235-3249, 1984.

[14] A. Suter, E. Morenzoni, N. Garifianov, R. Khasanov, E. Kirk, H. Luetkens, T. Prokscha, and M. Horisberger. Observation of nonexponential magnetic penetration profiles in the meissner state: A manifestation of nonlocal effects in superconductors. Phys. Rev. B, 72:024506, July 2005.

[15] V. F. Kozhevnikov, C. V. Giuraniuc, M. J. Van Bael, K. Temst, C. Van Haesendonck, T. M. Mishonov, T. Charlton, R. M. Dalgliesh, Yu. N. Khaidukov, Yu. V. Nikitenko, V. L. Aksenov, V. N. Gladilin, V. M. Fomin, J. T. Devreese, and J. O. Indekeu. Evidence for nonmonotonic magnetic field penetration in a type-I superconductor. Phys. Rev. B, 78:012502, July 2008.

[16] T. Van Cauteren and J. O. Indekeu. Non-local electrodynamics effect on surface superconductivity. EPL (Europhysics Letters), 94(4):46001, 2011.

[17] V. Kozhevnikov, A. Suter, H. Fritzsche, V. Gladilin, A. Volodin, T. Moorkens, M. Trekels, J. Cuppens, B. M. Wojek, T. Prokscha, E. Morenzoni, G. J. Nieuwenhuys, M. J. Van Bael, K. Temst, C. Van Haesendonck, and J. O. Indekeu. Nonlocal effect and dimensions of cooper pairs measured by low-energy muons and polarized neutrons in type-I superconductors. Phys. Rev. B, 87:104508, Mar 2013. 
[18] F. London and H. London. The electromagnetic equations of the supraconductor. Proceedings of the Royal Society of London. Series A, Mathematical and Physical Sciences, 149(866):71-88, 1935 .

[19] M. Fabrizio and A. Morro. Electromagnetism of Continuous Media. Oxford University Press Inc., New York, 2003.

[20] A. Alonso. A mathematical justification of the low-frequency heterogeneous time-harmonic Maxwell equations. Math. Models Methods Appl. Sci., 9(3):475-489, 1999.

[21] H. Ammari, A. Buffa, and J.-C Nédélec. A justification of eddy currents model for the maxwell equations. SIAM J. Appl. Math., 60(5):1805-1823, May 2000.

[22] P. Monk. Finite Element Methods for Maxwell's Equations. Oxford University Press Inc., New York, 2003.

[23] F.M. Odeh. On the integrodifferential equations of the nonlocal theory of superconductivity. J. Math. Phys., 1168(5), 1964.

[24] T. Lin and R.C. Rogers. Accurate computation of the field in pippard's nonlocal superconductivity model. Integral Equations and Applications, 7:167-192, 1995.

[25] A. Visintin. Maxwell's equations with vector hysteresis. Arch. Ration. Mech. Anal., 175(1):1-37, 2005.

[26] K. Van Bockstal and M. Slodička. The well-posedness of a nonlocal hyperbolic model for type-I superconductors. J. Mathematical Analysis and Applications, 2013. submitted.

[27] A. Bossavit. Computational electromagnetism. Variational formulations, complementarity, edge elements. Orlando, FL: Academic Press, 1998.

[28] A. Visintin. Electromagnetic processes in doubly-nonlinear composites. Commun. Partial Differ. Equations, 33(5):808-841, 2008.

[29] T. Roubíček and G. Tomassetti. Ferromagnets with eddy currents and pinning effects: their thermodynamics and analysis. Math. Models Methods Appl. Sci., 21(1):29-55, 2011.

[30] J. Kačur. Method of Rothe in evolution equations, volume 80 of Teubner Texte zur Mathematik. Teubner, Leipzig, 1985.

[31] G. Palatucci, O. Savin, and E. Valdinoci. Local and global minimizers for a variational energy involving a fractional norm. Annali di Matematica Pura ed Applicata, 2012. DOI: 10.1007/s10231011-0243-9.

[32] T. Roubíček. Nonlinear partial differential equations with applications, volume 153 of ISNM. Birkhäuser Verlag, Basel Boston Berlin, 2005.

[33] A. Kufner, O. John, and S. Fučík. Function Spaces. Monographs and textbooks on mechanics of solids and fluids. Noordhoff International Publishing, Leyden, 1977.

[34] J. Mercer. Functions of positive and negative type, and their connection with the theory of integral equations. Philosophical Transactions of the Royal Society of London. Series A, Containing Papers of a Mathematical or Physical Character, 209(441-458):415-446, 1909.

[35] G. Fasshauer. Solving differential equations with radial basis functions: multilevel methods and smoothing. Advances in Computational Mathematics, 11:139-159, 1999. 10.1023/A:1018919824891. 
[36] I. J. Schoenberg. Metric spaces and completely monotone functions. Ann. of Math., 39(4):811$841,1938$.

[37] C. Berg. Stieltjes-Pick-Bernstein-Schoenberg and their connection to complete monotonicity. In Mateu, J. and Porcu, E., Positive Definite Functions: from Schoenberg to Space-Time Challenges. Department of Mathematics, Universitat Jaume I, Castellon, Spain, 2008.

[38] E. W. Cheney and W. A. Light. A Course in Approximation Theory, volume 101 of Graduate Studies in Mathematics. American Mathematical Society, Providence, Rhode Island, 2000.

[39] L. C. Evans. Partial differential equations, volume 19 of Graduate Studies in Mathematics. American Mathematical Society, 1998.

[40] A. Logg, K-A. Mardal, G.N. Wells, et al. Automated Solution of Differential Equations by the Finite Element Method. Springer, 2012. 


\section{List of Figures}

1 The magnetic field inside a superconducting slab of thickness $2 L \ldots \ldots \ldots \ldots \ldots$

2 Results of numerical experiment 1 . (a) convergence rate for experiment 1 with $\widetilde{C}=2$. (b) convergence rate for experiment 1 with $\widetilde{C}=150 \ldots \ldots \ldots \ldots \ldots$

3 Results of numerical experiment 2. (a) convergence rate for experiment 2 with $\widetilde{C}=2$.

(b) convergence rate for experiment 2 with $\widetilde{C}=150 \ldots \ldots \ldots \ldots \ldots \ldots$

4 Results of numerical experiment 3. (a) convergence rate for experiment 3 with $\widetilde{C}=2$.

(b) convergence rate for experiment 3 with $\widetilde{C}=150 \ldots \ldots \ldots$ 
Table 1: $\left|\operatorname{Vol}(S)-\operatorname{Vol}\left(\mathcal{T}_{\boldsymbol{x}}\right)\right|$ for $10<n_{x} \leqslant 30$

\begin{tabular}{cccccccc}
$n_{x}$ & $\left|\operatorname{Vol}(S)-\operatorname{Vol}\left(\mathcal{T}_{\boldsymbol{x}}\right)\right|$ & $n_{x}$ & $\left|\operatorname{Vol}(S)-\operatorname{Vol}\left(\mathcal{T}_{\boldsymbol{x}}\right)\right|$ & $n_{x}$ & $\left|\operatorname{Vol}(S)-\operatorname{Vol}\left(\mathcal{T}_{\boldsymbol{x}}\right)\right|$ & $n_{x}$ & $\left|\operatorname{Vol}(S)-\operatorname{Vol}\left(\mathcal{T}_{\boldsymbol{x}}\right)\right|$ \\
\hline 11 & 0.000319 & 16 & 0.000206 & 21 & 0.000346 & 26 & 0.000312 \\
12 & 0.000441 & 17 & 0.000289 & 22 & 0.000244 & 27 & 0.000124 \\
13 & 0.000547 & 18 & 0.000416 & 23 & 0.000408 & 28 & 0.000271 \\
14 & 0.000184 & 19 & 0.000185 & 24 & 0.000138 & 29 & 0.000403 \\
15 & 0.000633 & 20 & 0.000561 & $\mathbf{2 5}$ & $\mathbf{0 . 0 0 0 0 9 3}$ & 30 & 0.000108
\end{tabular}

